\title{
KOSZUL DUALITY FOR STRATIFIED ALGEBRAS II. STANDARDLY STRATIFIED ALGEBRAS
}

\author{
VOLODYMYR MAZORCHUK
}

(Received 22 December 2008; accepted 2 June 2010)

Communicated by J. Du

\begin{abstract}
We give a complete picture of the interaction between the Koszul and Ringel dualities for graded standardly stratified algebras (in the sense of Cline, Parshall and Scott) admitting linear tilting (co)resolutions of standard and proper costandard modules. We single out a certain class of graded standardly stratified algebras, imposing the condition that standard filtrations of projective modules are finite, and develop a tilting theory for such algebras. Under the assumption on existence of linear tilting (co)resolutions we show that algebras from this class are Koszul, that both the Ringel and Koszul duals belong to the same class, and that these two dualities on this class commute.
\end{abstract}

2000 Mathematics subject classification: primary 16S37; secondary 16E30, 16G10.

Keywords and phrases: stratified algebra, Koszul algebra, tilting module, Ringel duality.

\section{Introduction}

In the theory of quasihereditary algebras there are two classical dualities: the Ringel duality, associated with the characteristic tilting module (see [Ri]), and the Koszul duality, associated with the category of linear complexes of projective modules (see [ADL1, CPS2, MO]). In [Ma, MO], it is shown that a certain class of Koszul quasihereditary algebras is stable with respect to taking both the Koszul and Ringel duals and that on this class of algebras the Koszul and Ringel dualities commute.

The approach of [Ma] is ultimately based on the possibility of realizing the derived category of our algebra as the homotopy category of complexes of tilting modules. This also suggested that the arguments of [Ma] should work in a much more general setup, whenever an appropriate stratification of the algebra and a sensible tilting theory with respect to this stratification exist. The aim of this paper is to define a setup for the study of the Koszul property of stratified algebras and to extend to this setup the main result of $[\mathrm{Ma}]$. We note that Koszul standardly stratified algebras that are not quasihereditary appear naturally in [ADL2, Fr3, KKM].

(C) 2010 Australian Mathematical Publishing Association Inc. 1446-7887/2010 \$16.00 
The most general setup for stratified algebras seems to be the notion of standardly stratified algebras, as introduced by Cline et al. [CPS1]. The main problem which one faces, trying to generalize [Ma] to such stratified algebras, is that, in general, standardly stratified algebras have infinite global dimension. In particular, this means that the Koszul dual of such an algebra (in the case when the original algebra is Koszul) is always infinite dimensional. Therefore any reasonable extension of [Ma] to stratified algebras must deal with infinite-dimensional stratified algebras, for which many of the classical results are not proved and lots of classical techniques are not developed.

In this paper, we study the class of positively graded standardly stratified algebras with finite-dimensional homogeneous components satisfying the additional assumption that all projective modules have finite standard filtrations. For such algebras we develop an analogue of the classical tilting theory and Ringel duality. This follows the classical theory closely; however, at some places, one has to be careful as we work with infinite-dimensional algebras, so some extension spaces might be infinite dimensional. We use the grading to split these infinite-dimensional spaces into an (infinite) direct sum of finite-dimensional ones. We also give some examples which justify our choice of algebras and show that outside the class we define, the classical approach to tilting theory fails. The Ringel duality functor turns out to be an antiequivalence between three different kinds of derived categories.

Using the standard grading of a characteristic tilting module, we restrict our attention to those standardly stratified algebras, for which all tilting coresolutions of standard modules and all tilting resolutions of proper costandard modules are linear. For an algebra $A$, let $R(A)$ and $E(A)$ denote the Ringel and Koszul duals of $A$, respectively. Generalizing the arguments of [Ma], we prove the following result (see Section 2 for the definitions).

THEOREM 1. Let A be a positively graded standardly stratified algebra with finitedimensional homogeneous components. Assume that:

(a) Every indecomposable projective A-module has a finite standard filtration.

(b) Every standard A-module has a linear tilting coresolution.

(c) Every costandard A-module has a linear tilting resolution.

Then the following results hold.

(i) The algebra A is Koszul.

(ii) The algebras $A, R(A), E(A), E(R(A))$ and $R(E(A))$ have properties $(a)$, (b) and $(c)$.

(iii) Every simple A-module is represented (in the derived category) by a linear complex of tilting modules.

(iv) $R(E(A)) \cong E(R(A))$ as graded standardly stratified algebras.

Theorem 1 extends and generalizes results from [ADL1, ADL2, Ma, MO].

The paper is organized as follows. In Section 2 we collect all necessary definitions and preliminaries. In Sections 3 and 4 we develop the tilting theory for graded standardly stratified algebras. This theory is used in Section 5 to prove Theorem 1 . We complete the paper with several examples in Section 6. 


\section{Graded standardly stratified algebras}

By $\mathbb{N}$ we denote the set of all positive integers. By a grading, we always mean a $\mathbb{Z}$-grading and by a module, we always mean a graded left module.

Let $\mathbb{k}$ be an algebraically closed field and $A=\bigoplus_{i \geq 0} A_{i}$ be a graded $\mathbb{k}$-algebra. We assume that $A$ is locally finite, that is, $\operatorname{dim}_{\mathbb{k}} A_{i}<\infty$. Set $\mathrm{r}(A):=\bigoplus_{i>0} A_{i}$. We further assume that $A_{0} \cong \bigoplus_{\lambda \in \Lambda} \mathbb{k} e_{\lambda}$ for some set $\left\{e_{\lambda}: \lambda \in \Lambda\right\}$ of pairwise orthogonal nonzero idempotents in $A_{0}$, where $\Lambda$ is a nonempty finite set (using the classical Morita theory, one extends all our results to the case when $A_{0}$ is a semisimple algebra). Under these assumptions, the algebra $A$ is positively graded in the sense of [MOS]. In what follows, we call $A$ positively graded if it satisfies all assumptions of this paragraph. A typical example of a positively graded algebra is $\mathbb{k}[x]$, where 1 has degree zero and $x$ has degree one.

Let $A$-gmod denote the category of all locally finite-dimensional graded $A$ modules. Morphisms in this category are homogeneous morphisms of degree zero between graded $A$-modules. Consider the full subcategories $A^{\uparrow}$-gmod and $A^{\downarrow}$-gmod of $A$-gmod that consist of all graded modules $M=\bigoplus_{i \in \mathbb{Z}} M_{i}$ for which there exists $n \in \mathbb{Z}$ such that $M_{i}=0$ for all $i>n$ or for all $i<n$, respectively. All these categories are abelian, the category $A^{\downarrow}$-gmod has enough projective modules and the category $A^{\uparrow}$-gmod has enough injective modules. For $M \in A^{\downarrow}$-gmod, we set

$$
\mathfrak{b}(M)= \begin{cases}+\infty & \text { if } M=0 \\ \min _{n \in \mathbb{Z}}\left\{M_{n} \neq 0\right\} & \text { if } M \neq 0\end{cases}
$$

For $i \in \mathbb{Z}$, we denote by $\langle i\rangle$ the autoequivalence of $A$-gmod that shifts the grading as follows: $(M\langle i\rangle)_{j}=M_{i+j}$, where $j \in \mathbb{Z}$. This autoequivalence preserves both $A^{\uparrow}$-gmod and $A^{\downarrow}$-gmod. Denote by $\circledast$ the usual graded duality on $A$-gmod (it swaps $A^{\uparrow}$-gmod and $A^{\downarrow}$-gmod). We adopt the notation $\operatorname{hom}_{A}$ and $\operatorname{ext}_{A}^{i}$ to denote homomorphisms and extensions in $A$-gmod. Unless stated otherwise, all morphisms are considered in the category $A$-gmod.

For $\lambda \in \Lambda$, we consider the graded indecomposable projective module $P(\lambda)=A e_{\lambda}$, its graded simple quotient $L(\lambda)=P(\lambda) / \mathrm{r}(A) P(\lambda)$ and the graded indecomposable injective envelope $I(\lambda)$ of $L(\lambda)$. Note that the following always hold: $P(\lambda) \in$ $A^{\downarrow}$-gmod, $I(\lambda) \in A^{\uparrow}$-gmod and $L(\lambda) \in A^{\downarrow}$-gmod $\cap A^{\uparrow}$-gmod.

Let $\preceq$ be a partial preorder on $\Lambda$. For $\lambda, \mu \in \Lambda$, we write $\lambda \prec \mu$ provided that $\lambda \preceq \mu$ and $\mu \npreceq \lambda$. We also write $\lambda \sim \mu$ provided that $\lambda \preceq \mu$ and $\mu \preceq \lambda$. Then $\sim$ is an equivalence relation. Let $\bar{\Lambda}$ denote the set of equivalence classes of $\sim$. Then the preorder $\preceq$ induces a partial order on $\bar{\Lambda}$, which we will denote by the same symbol, abusing notation. For $\lambda \in \Lambda$ we denote by $\bar{\lambda}$ the equivalence class in $\bar{\Lambda}$ containing $\lambda$. We also denote by $\preceq$ op the partial preorder on $\Lambda$, opposite to $\preceq$.

For $\lambda \in \Lambda$ we define the standard module $\Delta(\lambda)$ as the quotient of $P(\lambda)$ by the submodule generated by the images of all possible morphisms $P(\mu)\langle i\rangle \rightarrow P(\lambda)$, where $\lambda \prec \mu$ and $i \in \mathbb{Z}$. We also define the proper standard module $\bar{\Delta}(\lambda)$ as the quotient of $P(\lambda)$ by the submodule generated by the images of all possible 
morphisms $P(\mu)\langle i\rangle \rightarrow P(\lambda)$, where $\lambda \preceq \mu$ and $i \in \mathbb{Z}$ satisfies $i<0$. By definition, the modules $\Delta(\lambda)$ and $\bar{\Delta}(\lambda)$ belong to $A^{\downarrow}$-gmod. Dually we define the costandard module $\nabla(\lambda)$ and the proper costandard module $\bar{\nabla}(\lambda)$ (which always belong to $A^{\uparrow}$-gmod).

The algebra $A$ will be called standardly stratified (with respect to the preorder $\preceq$ on $\Lambda$ ) provided that for every $\lambda \in \Lambda$ the kernel $K(\lambda)$ of the canonical projection $P(\lambda) \rightarrow$ $\Delta(\lambda)$ has a finite filtration, whose subquotients are isomorphic (up to shift) to standard modules. This is a natural generalization of the original definition from [CPS1] to our setup. For example, the algebra $A$ is always standardly stratified (with projective standard modules) in the case when $|\Lambda|=1$ and, more generally, in the case when the relation $\preceq$ is the full relation.

\section{Tilting theory for graded standardly stratified algebras}

Tilting theory for (finite-dimensional) quasihereditary algebras was developed in [Ri]. It was extended in [AHLU] to (finite-dimensional) strongly standardly stratified algebras, and in [Fr2] to all (finite-dimensional) standardly stratified algebras. For infinite-dimensional algebras some versions of tilting theory appear in [CT, DM, MT]. This section is a further generalization of all these results, especially of those from [Fr2], to the case of infinite-dimensional positively graded algebras. In this section, $A$ is a positively graded standardly stratified algebra.

Let $\mathcal{C}(\Delta)$ denote the full subcategory of the category $A^{\downarrow}$-gmod that consists of all modules $M$ admitting a (possibly infinite) filtration

$$
M=M^{(0)} \supseteq M^{(1)} \supseteq M^{(2)} \supseteq \cdots,
$$

such that every subquotient $M^{(i)} / M^{(i+1)}$ (where $i=0,1, \ldots$ ) is isomorphic (up to shift) to some standard module and $\lim _{i \rightarrow+\infty} \mathfrak{b}\left(M^{(i)}\right)=+\infty$. Note that for $M \in A^{\downarrow}$-gmod with such a filtration we automatically get $\bigcap_{i \geq 0} M^{(i)}=0$. Denote by $\mathcal{F}^{\downarrow}(\Delta)$ the full subcategory of $A^{\downarrow}$-gmod that consists of all modules $M$ admitting a finite filtration with subquotients from $\mathcal{C}(\Delta)$. The category $\mathcal{F}^{\downarrow}(\Delta)$ is obviously closed with respect to finite extensions. Similarly we define $\mathcal{F}^{\downarrow}(\bar{\nabla})$. Let $\mathcal{F}^{b}(\Delta)$ and $\mathcal{F}^{b}(\bar{\nabla})$ be the corresponding full subcategories of modules with finite filtrations of the form (1). We start with the following result, which generalizes the corresponding results from $[\mathrm{AB}, \mathrm{AR}, \mathrm{Fr} 2$, Ri].

THEOREM 2. Let A be a positively graded standardly stratified algebra.

(i) We have

$$
\begin{aligned}
\mathcal{F}^{\downarrow}(\Delta) & =\left\{M \in A^{\downarrow}-\operatorname{gmod}: \operatorname{ext}_{A}^{i}(M, \bar{\nabla}(\lambda)\langle j\rangle)=0, \forall j \in \mathbb{Z}, i>0, \lambda \in \Lambda\right\} \\
& =\left\{M \in A^{\downarrow}-\operatorname{gmod}: \operatorname{ext}_{A}^{1}(M, \bar{\nabla}(\lambda)\langle j\rangle)=0, \forall j \in \mathbb{Z}, \lambda \in \Lambda\right\} .
\end{aligned}
$$

(ii) We have

$$
\begin{aligned}
\mathcal{F}^{\downarrow}(\bar{\nabla}) & =\left\{M \in A^{\downarrow} \text {-gmod : } \operatorname{ext}_{A}^{i}(\Delta(\lambda)\langle j\rangle, M)=0, \forall j \in \mathbb{Z}, i>0, \lambda \in \Lambda\right\} \\
& =\left\{M \in A^{\downarrow} \text {-gmod : } \operatorname{ext}_{A}^{1}(\Delta(\lambda)\langle j\rangle, M)=0, \forall j \in \mathbb{Z}, \lambda \in \Lambda\right\} .
\end{aligned}
$$


To prove Theorem 2 we will need several auxiliary lemmata. We will often use the usual induction for stratified algebras. To define this let $\lambda \in \Lambda$ be maximal with respect to $\preceq$. Set $e_{\bar{\lambda}}=\sum_{\mu \in \bar{\lambda}} e_{\mu}$ and $I_{\bar{\lambda}}=A e_{\bar{\lambda}} A$, and define $B_{\bar{\lambda}}=A / I_{\bar{\lambda}}$. The algebra $B_{\bar{\lambda}}$ inherits from $A$ a positive grading, and hence is a positively graded locally finite algebra. Further, as in the case of usual stratified algebras, the algebra $B_{\bar{\lambda}}$ is stratified with respect to the restriction of the preorder $\preceq$ to $\Lambda \backslash\{\bar{\lambda}\}$. Any module $M$ over $B_{\bar{\lambda}}$ can be considered as an $A$-module in the usual way. Set $P(\bar{\lambda})=\bigoplus_{\mu \in \bar{\lambda}} P(\mu)$.

LEMMA 3. For all $M, N \in B \frac{\downarrow}{\lambda}-\operatorname{gmod}$ and all $i \geq 0$,

$$
\operatorname{ext}_{B_{\bar{\lambda}}}^{i}(M, N)=\operatorname{ext}_{A}^{i}(M, N)
$$

PROOF. Let $\mathcal{P}^{\bullet}$ denote the minimal projective resolution of $M$ in $A^{\downarrow}$-gmod. Since $M \in B \frac{\downarrow}{\lambda}$-gmod, there exists $k \in \mathbb{Z}$ such that $M_{j}=0$ whenever $j<k$. As $A$ is positively graded, $\mathcal{P}_{j}^{i}=0$ for all $i$ and $j$ such that $j<k$.

Consider the projective module $P=\bigoplus_{j \leq-k} P(\bar{\lambda})\langle j\rangle$. As $A$ is standardly stratified, for every $i$ the sum $T^{i}$ of images of all homomorphisms from $P$ to $\mathcal{P}^{i}$ has the form $\bigoplus_{j \leq-k} P_{i, j}$, where $P_{i, j} \in$ add $P(\bar{\lambda})\langle j\rangle$.

The differential of $\mathcal{P}^{\bullet}$ obviously maps $T^{i}$ to $T^{i-1}$, which means that the sum of all $T^{i}$ is a subcomplex of $\mathcal{P}^{\bullet}$, call it $\mathcal{T} \bullet$. Since $M \in B \frac{\downarrow}{\lambda}$-gmod, the quotient $\overline{\mathcal{P}}^{\bullet}$ of $\mathcal{P}^{\bullet}$ by $\mathcal{T}^{\bullet}$ gives a minimal projective resolution of $M$ over $B_{\bar{\lambda}}$.

Since $N \in B \frac{\downarrow}{\lambda}$-gmod, any homomorphism from $\mathcal{P}^{i}$ to $N$ annihilates $\mathcal{T}^{i}$ and hence factors through $\overline{\mathcal{P}}^{i}$. The lemma follows.

LEMMA 4. For all $\mu \in \Lambda$ we have $\bar{\nabla}(\mu) \in A^{\downarrow}$-gmod, in particular, $\bar{\nabla}(\mu)$ is finite dimensional.

PROOF. We proceed by induction on the cardinality of $\bar{\Lambda}$. If $|\bar{\Lambda}|=1$, then all $\Delta(\lambda)$ are projective and all $\bar{\nabla}(\mu)$ are simple, so the claim is trivial.

Assume now that $|\bar{\Lambda}|>1$. Let $\lambda \in \Lambda$ be maximal. Then for all $\mu \notin \bar{\lambda}$, the claim follows from the inductive assumption applied to the stratified algebra $B_{\bar{\lambda}}$.

Assume, finally, that $\mu \in \bar{\lambda}$ is such that $\bar{\nabla}(\mu) \notin A^{\downarrow}$-gmod. Then there exists $v \in \Lambda$ and an infinite sequence of positive integers $0<j_{1}<j_{2}<\cdots$ such that for any $l \in \mathbb{N}$ there exists a nonzero homomorphism from $P(v)\left\langle j_{l}\right\rangle$ to $\bar{\nabla}(\mu)$. Let $M_{l}$ denote the image of this homomorphism. Then $M_{l}$ has simple top $L(v)\left\langle j_{l}\right\rangle$ and simple socle $L(\mu)$, and all other composition subquotients are of the form $L\left(v^{\prime}\right)\langle j\rangle$, where $v^{\prime} \prec \mu$ and $1 \leq j \leq j_{l}-1$.

The module $M_{l}\left\langle-j_{l}\right\rangle$ is thus a quotient of $P(\nu)$. Then the socle $L(\mu)\left\langle-j_{l}\right\rangle$ of $M_{l}\left\langle-j_{l}\right\rangle$ gives rise to a nonzero homomorphism from $P(\mu)\left\langle-j_{l}\right\rangle$ to $P(v)$. Since $\mu$ is maximal and all other composition subquotients of $M_{l}\left\langle-j_{l}\right\rangle$ are of the form $L\left(v^{\prime}\right)\langle j\rangle$ for some $\nu^{\prime} \prec \mu$, the above homomorphism gives rise to an occurrence of the standard module $\Delta(\mu)\left\langle-j_{l}\right\rangle$ in the standard filtration of $P(v)$. However, we have infinitely 
many $j_{l}$ and, at the same time, the standard filtration of $P(v)$ is finite. This is a contradiction, which yields the lemma.

LEMMA 5. For all $i, j \in \mathbb{Z}$ such that $i \geq 0$, and all $\lambda, \mu \in \Lambda$,

$$
\operatorname{ext}_{A}^{i}(\Delta(\lambda), \bar{\nabla}(\mu)\langle j\rangle)= \begin{cases}\mathbb{k} & \text { if } i=j=0 \text { and } \lambda=\mu, \\ 0 & \text { otherwise }\end{cases}
$$

PROOF. We proceed by induction on the cardinality of $\bar{\Lambda}$. If $|\bar{\Lambda}|=1$, then all $\Delta(\lambda)$ are projective and all $\bar{\nabla}(\mu)$ are simple, so the claim is trivial.

Assume now that $|\bar{\Lambda}|>1$. Let $\lambda^{\prime} \in \Lambda$ be maximal. Then, by definition, the module $\Delta(\lambda)$ is projective for all $\lambda \in \overline{\lambda^{\prime}}$. Hence for such $\lambda$ the lemma follows from the definition of $\bar{\nabla}(\mu)$. If $\lambda, \mu \notin \overline{\lambda^{\prime}}$, the claim follows from the inductive assumption applied to the standardly stratified algebra $B_{\overline{\lambda^{\prime}}}$ and Lemma 3.

Consider now the case when $\mu \in \overline{\lambda^{\prime}}$ and $\lambda \notin \overline{\lambda^{\prime}}$. Then $\Delta(\lambda)$ does not have any composition subquotient of the form $L(\mu)\langle j\rangle$ and hence

$$
\operatorname{hom}_{A}(\Delta(\lambda), \bar{\nabla}(\mu)\langle j\rangle)=0 .
$$

Let us check that

$$
\operatorname{ext}_{A}^{1}(\Delta(\lambda), \bar{\nabla}(\mu)\langle j\rangle)=0
$$

for all $j$. Applying $\operatorname{hom}_{A}\left(\Delta(\lambda),{ }_{-}\right)$to the short exact sequence

$$
\bar{\nabla}(\mu)\langle j\rangle \hookrightarrow I(\mu)\langle j\rangle \rightarrow \text { Coker },
$$

we obtain the exact sequence

$$
\operatorname{hom}_{A}(\Delta(\lambda), \text { Coker }) \rightarrow \operatorname{ext}_{A}^{1}(\Delta(\lambda), \bar{\nabla}(\mu)\langle j\rangle) \rightarrow \operatorname{ext}_{A}^{1}(\Delta(\lambda), I(\mu)\langle j\rangle) .
$$

Here the right-hand term is equal to zero by the injectivity of $I(\mu)$. By the definition of $\bar{\nabla}(\mu)$, the socle of Coker has (up to shift) only simple modules of the form $L(v)$, where $v \in \overline{\lambda^{\prime}}$, which implies that the left-hand term is equal to zero as well. The equality (2) follows.

Now we prove our claim by induction on $\lambda$ with respect to the preorder $\preceq$ (as mentioned above, the claim is true for $\lambda$ maximal). Apply $\operatorname{hom}_{A}(-, \bar{\nabla}(\mu)\langle j\rangle)$ to the short exact sequence

$$
\operatorname{Ker} \hookrightarrow P(\lambda) \rightarrow \Delta(\lambda)
$$

and, using the projectivity of $P(\lambda)$, obtain the following exact sequence:

$$
0 \rightarrow \operatorname{ext}_{A}^{i-1}(\operatorname{Ker}, \bar{\nabla}(\mu)\langle j\rangle) \rightarrow \operatorname{ext}_{A}^{i}(\Delta(\lambda), \bar{\nabla}(\mu)\langle j\rangle) \rightarrow 0
$$

for each $i>1$. Since $A$ is standardly stratified, Ker has a finite filtration by standard modules of the form $\Delta(v)$, where $\lambda \prec v$, (up to shift). Hence, from the inductive assumption, $\operatorname{ext}_{A}^{i-1}(\operatorname{Ker}, \bar{\nabla}(\mu)\langle j\rangle)=0$. This shows that $\operatorname{ext}_{A}^{i}(\Delta(\lambda), \bar{\nabla}(\mu)\langle j\rangle)=0$ and completes the proof. 
COROLlARY 6. Let A be a positively graded standardly stratified algebra.

(i) For any $M \in \mathcal{F}^{\downarrow}(\Delta), \lambda \in \Lambda, i \in \mathbb{N}$ and $j \in \mathbb{Z}$,

$$
\operatorname{ext}^{i}(M, \bar{\nabla}(\lambda)\langle j\rangle)=0 .
$$

(ii) For any $M \in \mathcal{F}^{\downarrow}(\bar{\nabla}), \lambda \in \Lambda, i \in \mathbb{N}$ and $j \in \mathbb{Z}$,

$$
\operatorname{ext}^{i}(\Delta(\lambda)\langle j\rangle, M)=0 .
$$

PROOF. It is certainly enough to prove statement (i) in the case when $M$ has a filtration of the form (1). As $\lim _{i \rightarrow+\infty} \mathfrak{b}\left(M^{(i)}\right)=+\infty$ and $\bar{\nabla}(\lambda)$ is finite dimensional by Lemma 4 , there exists $n \in \mathbb{Z}$ such that for any $i \in \mathbb{Z}$ with $\bar{\nabla}(\lambda)\langle j\rangle_{i} \neq 0$ we have $i<\mathfrak{b}\left(M^{(n)}\right)$. Since $A$ is positively graded, there are no homomorphisms from any component of the projective resolution of $M^{(n)}$ to $\bar{\nabla}(\lambda)\langle j\rangle$. This means that all extensions from $M^{(n)}$ to $\bar{\nabla}(\lambda)\langle j\rangle$ vanish. At the same time, the quotient $M / M^{(n)}$ has a finite filtration by standard modules, and hence all extensions from $M / M^{(n)}$ to $\bar{\nabla}(\lambda)\langle j\rangle$ vanish by Lemma 5 . Statement (i) follows.

It is certainly enough to prove statement (ii) in the case when $M$ has a filtration of the form (1) (with subquotients being proper costandard modules). Let $\mathcal{P}^{\bullet}$ be the minimal projective resolution of $\Delta(\lambda)\langle j\rangle$. As every indecomposable projective has a finite standard filtration, it follows that $\mathcal{P}^{\bullet}$ has only finitely many nonzero components, and moreover, each of them is a finite direct sum of projective modules. As $\lim _{i \rightarrow+\infty} \mathfrak{b}\left(M^{(i)}\right)=+\infty$, there exists $n \in \mathbb{N}$ such that there are no maps from any $\mathcal{P}^{i}$ to $M^{(n)}$, in particular, all extensions from $\Delta(\lambda)\langle j\rangle$ to $M^{(n)}$ vanish. At the same time, the quotient $M / M^{(n)}$ has a finite filtration by proper costandard modules and hence all extensions from $\Delta(\lambda)\langle j\rangle$ to $M / M^{(n)}$ vanish by Lemma 5. Statement (ii) follows and the proof is complete.

The following lemma is just an observation that the category $\mathcal{F}^{\downarrow}(\bar{\nabla})$ can, in fact, be defined in a somewhat easier way than the one we used. For the category $\mathcal{F} \downarrow(\Delta)$ this is not possible in the general case, see Example 43.

LEMMA 7. Any module from $\mathcal{F}^{\downarrow}(\bar{\nabla})$ has a filtration of the form (1).

ProOF. Let $X, Z \in \mathcal{C}$ and

$$
X=X^{(0)} \supseteq X^{(1)} \supseteq X^{(2)} \supseteq \cdots,
$$

and

$$
Z=Z^{(0)} \supseteq Z^{(1)} \supseteq Z^{(2)} \supseteq \cdots,
$$

be filtrations of the form (1). Assume that $Y \in A^{\downarrow}$-gmod is such that there is a short exact sequence

$$
0 \rightarrow X \rightarrow Y \rightarrow Z \rightarrow 0
$$

To prove the lemma, it is enough to show that $Y$ has a filtration of the form (1). 
Since all costandard modules are finite dimensional by Lemma 4, and $\lim _{i \rightarrow+\infty} \mathfrak{b}\left(Z^{(i)}\right)=+\infty$, there exists $k \in\{0,1,2, \ldots\}$ such that $i<\mathbf{b}\left(Z^{(k)}\right)$ for any $i \in \mathbb{Z}$ with $\left(X^{(0)} / X^{(1)}\right)_{i} \neq 0$.

Now for $i=0,1, \ldots, k$, we let $Y^{(i)}$ be the full preimage of $Z^{(i)}$ in $Y$ under the projection $Y \rightarrow Z$. In this way, we get the first part of the filtration of $Y$ with proper costandard subquotients. On the next step, we let $Y^{(k+1)}$ denote the submodule of $Y^{(k)}$ generated by $X^{(1)}$ and $Y_{i}^{(k)}$, where $i \geq \mathbf{b}\left(Z^{(k)}\right)$. Then $Y^{(k+1)}+X^{(0)}=Y^{(k)}$ by construction. At the same time, from our choice of $k$ in the previous paragraph it follows that $Y^{(k+1)} \cap X^{(0)}=X^{(1)}$ and hence

$$
Y^{(k)} / Y^{(k+1)} \cong X^{(0)} / X^{(1)},
$$

which is a proper costandard module.

Now we proceed in the same way constructing a proper costandard filtration for $Y^{(k+1)}$. The condition $\lim _{i \rightarrow+\infty} \mathfrak{b}\left(Y^{(i)}\right)=+\infty$ follows from the construction. This completes the proof.

LEMMA 8. Let $M \in A^{\downarrow}$-gmod be such that $\operatorname{ext}_{A}^{1}(\Delta(\lambda)\langle j\rangle, M)=0$ for all $\lambda$ and $j$. Then $M \in \mathcal{F}^{\downarrow}(\bar{\nabla})$.

PROOF. First let us show that the conditions of the lemma imply that

$$
\operatorname{ext}_{A}^{i}(\Delta(\lambda)\langle j\rangle, M)=0
$$

for all $j$, all $\lambda$ and all $i>0$. If $\lambda$ is maximal, then the corresponding $\Delta(\lambda)$ is projective, and the claim is clear. Otherwise, we proceed by induction with respect to the preorder $\preceq$. We apply $\operatorname{hom}_{A}(-, M)$ to the short exact sequence (3), and the equality (4) follows from the inductive assumption by the dimension shift in the resulting long exact sequence.

We proceed by induction on the cardinality of $\bar{\Lambda}$. If $|\bar{\Lambda}|=1$, then $\mathcal{F} \downarrow(\bar{\nabla})=$ $A^{\downarrow}$-gmod, and the claim is trivial.

Assume now that $|\bar{\Lambda}|>1$, and let $\lambda^{\prime} \in \Lambda$ be maximal. Let $N$ denote the maximal submodule of $M$ that does not contain any composition factors of the form $L(\mu)$, where $\mu \in \overline{\lambda^{\prime}}$ (up to shift). Let $v \notin \overline{\lambda^{\prime}}$. Applying $\operatorname{hom}_{A}\left(\Delta(\nu)\langle j\rangle,{ }_{-}\right)$to the short exact sequence

$$
N \hookrightarrow M \rightarrow \text { Coker }
$$

we obtain the exact sequence

$$
\operatorname{hom}_{A}(\Delta(v)\langle j\rangle, \text { Coker }) \rightarrow \operatorname{ext}_{A}^{1}(\Delta(v)\langle j\rangle, N) \rightarrow \operatorname{ext}_{A}^{1}(\Delta(v)\langle j\rangle, M) .
$$

Here the right-hand term is zero by our assumptions, and the left-hand term is zero by the definition of $N$. This implies that the middle term is zero, which shows that $\operatorname{ext}_{B_{\overline{\lambda^{\prime}}}}^{1}(\Delta(v)\langle j\rangle, N)=0$, by Lemma 3. Applying the inductive assumption to the standardly stratified algebra $B_{\overline{\lambda^{\prime}}}$, we deduce that $N \in \mathcal{F}^{\downarrow}(\bar{\nabla})$. 
Since $\mathcal{F}^{\downarrow}(\bar{\nabla})$ is extension closed, to complete the proof we are left to show that Coker $\in \mathcal{F}^{\downarrow}(\bar{\nabla})$. Applying $\operatorname{hom}_{A}\left(\Delta(\lambda)\langle j\rangle,{ }_{-}\right)$to (5) and using (4), the previous paragraph and Lemma 5, we conclude that

$$
\operatorname{ext}_{A}^{i}(\Delta(\lambda)\langle j\rangle, \text { Coker })=0
$$

for all $j, \lambda$ and $i>0$.

If Coker $=0$, we are done. Otherwise, there exists some $\mu \in \overline{\lambda^{\prime}}$ and a maximal $j^{\prime} \in \mathbb{Z}$ such that there is a nonzero homomorphism from Coker to $I(\mu)\left\langle j^{\prime}\right\rangle$. Let $K$ denote the image of this homomorphism. Applying $\operatorname{hom}_{A}\left(\Delta(\lambda)\langle j\rangle,{ }_{-}\right)$to the short exact sequence

$$
\text { Ker } \hookrightarrow \text { Coker } \rightarrow K,
$$

and using the definition of $K$, we obtain

$$
\operatorname{ext}_{A}^{1}(\Delta(\lambda)\langle j\rangle, \text { Ker })=0
$$

for all $\lambda$ and $j$. The equality (8), the corresponding equalities (4) (for $M=$ Ker) and the dimension shift with respect to (7) then imply that

$$
\operatorname{ext}_{A}^{1}(\Delta(\lambda)\langle j\rangle, K)=0
$$

for all $\lambda$ and $j$.

By the definition of $K$, there is a short exact sequence

$$
K \hookrightarrow \bar{\nabla}(\mu)\left\langle j^{\prime}\right\rangle \rightarrow C^{\prime}
$$

for some cokernel $C^{\prime}$. By the definition of $\bar{\nabla}(\mu)$, all composition subquotients of $C^{\prime}$ have the form $L(\nu)$, where $v \prec \mu$ (up to shift). Let $\lambda \in \Lambda$ be such that $\lambda \prec \mu$. Applying $\operatorname{hom}_{A}\left(\Delta(\lambda)\langle j\rangle,{ }_{-}\right)$to (10), we get the exact sequence

$$
\operatorname{hom}_{A}\left(\Delta(\lambda)\langle j\rangle, \bar{\nabla}(\mu)\left\langle j^{\prime}\right\rangle\right) \rightarrow \operatorname{hom}_{A}\left(\Delta(\lambda)\langle j\rangle, C^{\prime}\right) \rightarrow \operatorname{ext}_{A}^{1}(\Delta(\lambda)\langle j\rangle, K) .
$$

Here the left-hand term is zero by the definition of $\bar{\nabla}(\mu)$ and the right-hand term is zero by (9). This shows that the middle term is zero as well and thus $C^{\prime}=0$, that is, $K$ is a proper costandard module.

We can now apply the same arguments as above to the module Ker in place of Coker and get the short exact sequence

$$
\operatorname{Ker}^{\prime} \hookrightarrow \operatorname{Ker} \rightarrow K^{\prime},
$$

where $K^{\prime}$ is proper costandard and $\operatorname{ext}_{A}^{1}\left(\Delta(\lambda)\langle j\rangle, \operatorname{Ker}^{\prime}\right)=0$ for all $\lambda$ and $j$. Proceeding inductively, we obtain a (possibly infinite) decreasing filtration

$$
\text { Coker } \supseteq \operatorname{Ker} \supseteq \operatorname{Ker}^{\prime} \supseteq \cdots
$$

with proper costandard subquotients. That $\lim _{i \rightarrow+\infty} \mathfrak{b}\left(\right.$ Coker $\left.^{(i)}\right)=+\infty$ follows from the construction, since all our modules are from $A^{\downarrow}$-gmod, all proper costandard modules (subquotients of the filtration of Coker) are finite dimensional by Lemma 4, and there are only finitely many proper costandard modules up to isomorphism and shift (which implies that the dimensions of proper costandard modules are uniformly bounded). Therefore Coker $\in \mathcal{F}^{\downarrow}(\bar{\nabla})$. The lemma follows. 
LEMMA 9. Let $M \in A^{\downarrow}$-gmod be such that $\operatorname{ext}_{A}^{1}(M, \bar{\nabla}(\mu)\langle j\rangle)=0$ for all $\mu$ and $j$. Then $M \in \mathcal{F}^{\downarrow}(\Delta)$.

PROOF. Let $M \in A^{\downarrow}$-gmod be such that $\operatorname{ext}_{A}^{1}(M, \bar{\nabla}(\mu)\langle j\rangle)=0$ for all $\mu$ and $j$. We again proceed by induction on $|\bar{\Lambda}|$. If $|\bar{\Lambda}|=1$, then proper costandard modules are simple and hence $M$ is projective. All indecomposable projective modules belong to $\mathcal{F}^{\downarrow}(\Delta)$ as $A$ is standardly stratified. Using this it is easy to check that all projective modules in $A^{\downarrow}$-gmod belong to $\mathcal{F}^{\downarrow}(\Delta)$. Hence, in the case when $|\bar{\Lambda}|=1$, the lemma is true.

If $|\bar{\Lambda}|>1$, we take some maximal $v \in \Lambda$ and denote by $N$ the sum of all images of all possible homomorphisms from $\Delta(\lambda)\langle j\rangle$, where $\lambda \in \bar{v}$ and $j \in \mathbb{Z}$, to $M$. Then there is a short exact sequence

$$
N \hookrightarrow M \rightarrow \text { Coker. }
$$

Compare this with (5) in the proof of Lemma 8. Using arguments similar to those in the latter proof, one shows that $\operatorname{ext}_{A}^{1}(\operatorname{Coker}, \bar{\nabla}(\mu)\langle j\rangle)=0$ for all $\mu \in \Lambda \backslash \bar{v}$ and all $j$. By construction, Coker is in fact a $B_{\bar{v}}$-module. Therefore, using Lemma 3 and the inductive assumption, Coker $\in \mathcal{F}^{\downarrow}(\Delta)$. From Corollary 6(i),

$$
\operatorname{ext}_{A}^{i}(\text { Coker, } \bar{\nabla}(\mu)\langle j\rangle)=0
$$

for all $\mu \in \Lambda, j \in \mathbb{Z}$ and $i \in \mathbb{N}$.

Furthermore, for any $\mu$ and $j$, we also have the following part of the long exact sequence associated with (12):

$$
\operatorname{ext}_{A}^{1}(M, \bar{\nabla}(\mu)\langle j\rangle) \rightarrow \operatorname{ext}_{A}^{1}(N, \bar{\nabla}(\mu)\langle j\rangle) \rightarrow \operatorname{ext}_{A}^{2}(\text { Coker, } \bar{\nabla}(\mu)\langle j\rangle) .
$$

The left-hand term is zero by our assumptions and the right-hand term is zero by (13). Therefore, for all $\mu$ and $j$,

$$
\operatorname{ext}_{A}^{1}(N, \bar{\nabla}(\mu)\langle j\rangle)=0 .
$$

Fix now $\mu \in \Lambda$ and $j \in \mathbb{Z}$ and denote by $C$ the cokernel of the natural inclusion $L(\mu)\langle j\rangle \hookrightarrow \bar{\nabla}(\mu)\langle j\rangle$. Applying $\operatorname{hom}_{A}\left(N,{ }_{-}\right)$to the short exact sequence

$$
L(\mu)\langle j\rangle \hookrightarrow \bar{\nabla}(\mu)\langle j\rangle \rightarrow C,
$$

and using (14) and the fact that $\operatorname{hom}_{A}(N, C)=0$ by construction, we see that $\operatorname{ext}_{A}^{1}(N, L(\mu)\langle j\rangle)=0$ for any $\mu$ and $j$. This shows that $N$ is projective and thus belongs to $\mathcal{F}^{\downarrow}(\Delta)$. Since $\mathcal{F}^{\downarrow}(\Delta)$ is closed under extensions, the lemma follows.

\section{Proof of Theorem 2. Define}

$$
\begin{aligned}
& \mathcal{X}=\left\{M \in A^{\downarrow} \text {-gmod : } \operatorname{ext}_{A}^{i}(M, \bar{\nabla}(\lambda)\langle j\rangle)=0, \forall j \in \mathbb{Z}, i>0, \lambda \in \Lambda\right\}, \\
& \mathcal{Y}=\left\{M \in A^{\downarrow} \text {-gmod : } \operatorname{ext}_{A}^{1}(M, \bar{\nabla}(\lambda)\langle j\rangle)=0, \forall j \in \mathbb{Z}, \lambda \in \Lambda\right\} .
\end{aligned}
$$

The inclusion $\mathcal{X} \subseteq \mathcal{Y}$ is obvious. The inclusion $\mathcal{Y} \subseteq \mathcal{F}^{\downarrow}(\Delta)$ follows from Lemma 9. The inclusion $\mathcal{F}^{\downarrow}(\Delta) \subseteq \mathcal{X}$ follows from Corollary 6(i). This proves Theorem 2(i). Theorem 2(ii) is proved similarly using Lemma 8 instead of Lemma 9 and Corollary 6(ii) instead of Corollary 6(i). 
COROLLARY 10. Let A be a positively graded standardly stratified algebra.

(i) For every $M \in \mathcal{F}^{\downarrow}(\Delta), \quad \lambda \in \Lambda$ and $j \in \mathbb{Z}$, the multiplicity of $\Delta(\lambda)\langle j\rangle$ in any standard filtration of $M$ is well-defined, finite and is equal to $\operatorname{dim}_{\operatorname{hom}_{A}}(M, \bar{\nabla}(\lambda)\langle j\rangle)$.

(ii) For every $M \in \mathcal{F}^{\downarrow}(\bar{\nabla}), \lambda \in \Lambda$ and $j \in \mathbb{Z}$, the multiplicity of $\bar{\nabla}(\lambda)\langle j\rangle$ in any proper costandard filtration of $M$ is well-defined, finite and is equal to $\operatorname{dim} \operatorname{hom}_{A}(\Delta(\lambda)\langle j\rangle, M)$.

PROOF. The corollary follows from Lemma 5 by standard arguments (see, for instance, [Ri]).

REMARK 11 . Note that the ungraded multiplicity of $\Delta(\lambda)$ (or $\bar{\nabla}(\lambda)$ ) in $M$ might be infinite.

Let $\mathcal{F}^{\uparrow}(\bar{\nabla})$ denote the full subcategory of the category $A^{\uparrow}$-gmod that consists of all modules $M$ admitting a (possibly infinite) filtration

$$
0=M^{(0)} \subseteq M^{(1)} \subseteq M^{(2)} \subseteq \cdots,
$$

such that $M=\bigcup_{i \geq 0} M^{(i)}$ and the subquotient $M^{(i+1)} / M^{(i)}$ is isomorphic (up to shift) to some proper costandard module for every $i=0,1, \ldots$ Since all proper costandard modules are finite dimensional by Lemma 4, from the dual version of Lemma 7 one deduces that $\mathcal{F}^{\uparrow}(\bar{\nabla})$ is closed under finite extensions.

THEOREM 12. We have

$$
\begin{aligned}
\mathcal{F}^{\uparrow}(\bar{\nabla}) & =\left\{M \in A^{\uparrow}-\operatorname{gmod}: \operatorname{ext}_{A}^{i}(\Delta(\lambda)\langle j\rangle, M)=0, \forall j \in \mathbb{Z}, i>0, \lambda \in \Lambda\right\} \\
& =\left\{M \in A^{\uparrow}-\operatorname{gmod}: \operatorname{ext}_{A}^{1}(\Delta(\lambda)\langle j\rangle, M)=0, \forall j \in \mathbb{Z}, \lambda \in \Lambda\right\} .
\end{aligned}
$$

ProOF. Set

$$
\begin{aligned}
& \mathcal{X}=\left\{M \in A^{\uparrow} \text {-gmod : } \operatorname{ext}_{A}^{1}(\Delta(\lambda)\langle j\rangle, M)=0, \forall j \in \mathbb{Z}, \lambda \in \Lambda\right\}, \\
& \mathcal{Y}=\left\{M \in A^{\uparrow} \text {-gmod : } \operatorname{ext}_{A}^{i}(\Delta(\lambda)\langle j\rangle, M)=0, \forall j \in \mathbb{Z}, i>0, \lambda \in \Lambda\right\} .
\end{aligned}
$$

Obviously, $\mathcal{Y} \subseteq \mathcal{X}$.

Let $M \in \mathcal{F}^{\uparrow}(\bar{\nabla}), \lambda \in \Lambda$ and $j \in \mathbb{Z}$. Assume that (15) gives a proper costandard filtration of $M$. As $M \in A^{\uparrow}$-gmod and $\Delta(\lambda) \in A^{\downarrow}$-gmod, it follows that there exists $k \in \mathbb{N}$ such that

$$
\operatorname{ext}_{A}^{i}\left(\Delta(\lambda)\langle j\rangle, M / M^{(k)}\right)=0
$$

for all $i \geq 0$. At the same time,

$$
\operatorname{ext}_{A}^{i}\left(\Delta(\lambda)\langle j\rangle, M^{(k)}\right)=0
$$

for all $i>0$, by Lemma 5. Hence

$$
\operatorname{ext}_{A}^{i}(\Delta(\lambda)\langle j\rangle, M)=0
$$

for all $i>0$, and thus $\mathcal{F}^{\uparrow}(\bar{\nabla}) \subseteq \mathcal{Y}$. 
It remains to show that $\mathcal{X} \subseteq \mathcal{F}^{\uparrow}(\bar{\nabla})$. We will do this by induction on $|\bar{\Lambda}|$. If $|\bar{\Lambda}|=1$, then all proper standard modules are simple, which shows that $\mathcal{F}^{\uparrow}(\bar{\nabla})=A^{\uparrow}$-gmod. In this case, the inclusion $\mathcal{X} \subseteq \mathcal{F}^{\uparrow}(\bar{\nabla})$ is obvious.

If $|\bar{\Lambda}|>1$, then we fix some maximal $\mu \in \Lambda$. Let $M \in \mathcal{X}$. Denote by $N$ the maximal submodule of $M$ satisfying $[N: L(\nu)\langle j\rangle]=0$ for all $v \in \bar{\mu}$ and $j \in \mathbb{Z}$. For $\lambda \in \Lambda$ and $j \in \mathbb{Z}$, applying the functor $\operatorname{hom}_{A}\left(\Delta(\lambda)\langle j\rangle,{ }_{-}\right)$to the short exact sequence

$$
N \hookrightarrow M \rightarrow \text { Coker }
$$

and using the fact that $M \in \mathcal{X}$, gives the following exact sequences:

$$
\operatorname{hom}_{A}(\Delta(\lambda)\langle j\rangle, \text { Coker }) \rightarrow \operatorname{ext}_{A}^{1}(\Delta(\lambda)\langle j\rangle, N) \rightarrow 0
$$

and

$$
0 \rightarrow \operatorname{ext}_{A}^{1}(\Delta(\lambda)\langle j\rangle, \text { Coker }) \rightarrow \operatorname{ext}_{A}^{2}(\Delta(\lambda)\langle j\rangle, N)
$$

By construction, any simple subquotient in the socle of Coker has the form $L(\nu)\langle j\rangle$, for some $v \in \bar{\mu}$ and $j \in \mathbb{Z}$. Therefore, since $\mu$ is maximal, if $\lambda \notin \bar{\mu}$, then $\operatorname{hom}_{A}(\Delta(\lambda)\langle j\rangle$, Coker $)=0$ and hence $\operatorname{ext}_{A}^{1}(\Delta(\lambda)\langle j\rangle, N)=0$ from (16). For $\lambda \in \bar{\mu}$ the module $\Delta(\lambda)\langle j\rangle$ is projective and hence $\operatorname{ext}_{A}^{1}(\Delta(\lambda)\langle j\rangle, N)=0$ as well. This implies that $N \in \mathcal{X}$. As $N \in B_{\bar{\mu}}$-mod by construction, by using Lemma 3 and the inductive assumption, we deduce that $N \in \mathcal{F}^{\uparrow}(\bar{\nabla})$. As the inclusion $\mathcal{F}^{\uparrow}(\bar{\nabla}) \subseteq \mathcal{Y}$ is already proved, $N \in \mathcal{Y}$ and from (17) it follows that Coker $\in \mathcal{X}$.

Since $\mathcal{F}^{\uparrow}(\bar{\nabla})$ is closed under finite extensions, it remains to show that Coker $\epsilon$ $\mathcal{F}^{\uparrow}(\bar{\nabla})$. If Coker $=0$, there is nothing to do. If Coker $\neq 0$, we choose the maximal $k \in \mathbb{Z}$ such that Coker $_{k} \neq 0$. Denote by $V$ the intersection of the kernels of all possible maps from Coker to $I(\nu)\langle j\rangle$, where $v \in \bar{\mu}$ and $-j<k$, and consider the corresponding short exact sequence

$$
V \hookrightarrow \text { Coker } \rightarrow \text { Coker' }
$$

From the construction, it follows that the socle of $V$ is $V_{k}$, and that, for any $j<k$, every composition subquotient of $V_{j}$ has the form $L(v)\langle-j\rangle$ for some $v \notin \bar{\mu}$. Therefore, taking the injective envelope of $V$ and using the definition of proper standard modules, we deduce that $V$ is a submodule of a finite direct sum of proper standard modules (such that the socles of $V$ and of this direct sum agree). In particular, $V$ is finite dimensional, as both $V_{k}$ and all proper standard modules are, by Lemma 4 . Hence $V \in A^{\downarrow}$-gmod.

For $\lambda \in \Lambda$ and $j \in \mathbb{Z}$, applying the functor $\operatorname{hom}_{A}\left(\Delta(\lambda)\langle j\rangle,{ }_{-}\right)$to (18) and using Coker $\in \mathcal{X}$ gives the following exact sequences:

$$
\operatorname{hom}_{A}\left(\Delta(\lambda)\langle j\rangle, \text { Coker' }^{\prime}\right) \operatorname{ext}_{A}^{1}(\Delta(\lambda)\langle j\rangle, V) \rightarrow 0
$$

and

$$
0 \rightarrow \operatorname{ext}_{A}^{1}\left(\Delta(\lambda)\langle j\rangle, \text { Coker' }^{\prime}\right) \rightarrow \operatorname{ext}_{A}^{2}(\Delta(\lambda)\langle j\rangle, V) .
$$

If $\lambda \notin \bar{\mu}$, then $\operatorname{hom}_{A}\left(\Delta(\lambda)\langle j\rangle\right.$, Coker' $\left.^{\prime}\right)=0$, by the definition of the module Coker', and hence $\operatorname{ext}_{A}^{1}(\Delta(\lambda)\langle j\rangle, V)=0$ from (19). If $\lambda \in \bar{\mu}$, then $\Delta(\lambda)\langle j\rangle$ is projective by 
the maximality of $\mu$, and $\operatorname{ext}_{A}^{1}(\Delta(\lambda)\langle j\rangle, V)=0$ automatically. Hence $V \in \mathcal{X}$. Since $V \in A^{\downarrow}$-gmod as shown above, from Theorem 2(ii), we deduce that $V$ has a (finite) proper standard filtration and thus $V \in \mathcal{F}^{\uparrow}(\bar{\nabla})$. Using the inclusion $\mathcal{F}^{\uparrow}(\bar{\nabla}) \subseteq \mathcal{Y}$ (already proved) and (20), we also get Coker $^{\prime} \in \mathcal{X}$. Note that $\operatorname{Coker}_{k}^{\prime}=0$ by construction.

Applying now the same arguments to Coker' and proceeding inductively (decreasing $k$ ), we construct a (possibly infinite) proper costandard filtration of Coker' of the form (15). The theorem follows.

The following corollary is a weak version of [D1, Lemma 2.1] and [Fr2, Theorem 1]. The original statement also contains the converse assertion that the fact that indecomposable injective $A$-modules belong to $\mathcal{F}^{\uparrow}(\bar{\nabla})$ guarantees that $A$ is standardly stratified.

COROllary 13 (Weak version of Dlab's theorem). All indecomposable injective Amodules belong to $\mathcal{F}^{\uparrow}(\bar{\nabla})$.

PROOF. If $I$ is an indecomposable injective $A$-module, then obviously $\operatorname{ext}_{A}^{i}(\Delta(\lambda)\langle j\rangle$, $I)=0$ for all $j \in \mathbb{Z}, i>0$ and $\lambda \in \Lambda$, so the claim follows from Theorem 12 .

The following result generalizes the corresponding results of [AHLU, Fr2, Ri].

THEOREM 14 (Construction of tilting modules). Let $A$ be a positively graded standardly stratified algebra.

(i) The category $\mathcal{F}^{\downarrow}(\Delta) \cap \mathcal{F}^{\downarrow}(\bar{\nabla})$ is closed with respect to taking direct sums and direct summands.

(ii) For all $\lambda \in \Lambda$, there is a unique indecomposable object $T(\lambda) \in \mathcal{F}^{\downarrow}(\Delta) \cap \mathcal{F}^{\downarrow}(\bar{\nabla})$ for which there is a short exact sequence

$$
\Delta(\lambda) \hookrightarrow T(\lambda) \rightarrow \text { Coker }
$$

with Coker $\in \mathcal{F}^{\downarrow}(\Delta)$.

(iii) Every indecomposable object in $\mathcal{F}^{\downarrow}(\Delta) \cap \mathcal{F}^{\downarrow}(\bar{\nabla})$ has the form $T(\lambda)\langle j\rangle$ for some $\lambda \in \Lambda$ and $j \in \mathbb{Z}$.

We will need the following lemmata.

LEMMA 15. For all $\lambda, \mu \in \Lambda$, all $i \geq 0$ and all sufficiently large positive $j$,

$$
\operatorname{ext}_{A}^{i}(\Delta(\lambda)\langle j\rangle, \Delta(\mu))=0 .
$$

PROOF. We proceed by induction with respect to $\preceq$. If $\lambda$ is maximal, then the module $\Delta(\lambda)$ is projective and the claim is trivial when $i>0$. When $i=0$, the claim follows from the fact that $A$ is positively graded. Now, if $\lambda$ is not maximal, we consider the short exact sequence (3). In this sequence, Ker has a finite filtration by (shifted) standard modules, whose indices are strictly greater than $\lambda$ with respect to $\preceq$. Hence the claim follows by the usual dimension shift (note that it is enough to consider only finitely many values of $i$, namely, $i \leq|\Lambda|$ ). 
LEMMA 16. For all $\lambda, \mu \in \Lambda$ and $j \in \mathbb{Z}$, the inequality

$$
\operatorname{ext}_{A}^{1}(\Delta(\lambda)\langle j\rangle, \Delta(\mu)) \neq 0
$$

implies that $\lambda \prec \mu$.

PROOF. If $\lambda \nprec \mu$, then, using Lemma 3 , we may assume that $\lambda$ is maximal. In this case, $\Delta(\lambda)$ is projective and the claim becomes trivial.

Lemma 17. For all $M \in \mathcal{F}^{\downarrow}(\Delta), N \in \mathcal{F}^{\downarrow}(\bar{\nabla})$ and $i \in \mathbb{N}$,

$$
\operatorname{ext}_{A}^{i}(M, N)=0 \text {. }
$$

PROOF. It is enough to prove the claim in the case when $M$ has a filtration of the form (1). Let $\lambda$ be a maximal index occurring in standard subquotients of $M$. Then from Lemma 16, no corresponding standard subquotient extends any other standard subquotient of $M$. Therefore $M$ has a submodule isomorphic to a direct sum of shifted $\Delta(\lambda)$ such that the cokernel has a standard filtration in which no subquotient of the form $\Delta(\lambda)$ (up to shift) occur. Since $\Lambda$ is finite, proceeding inductively, we construct a finite filtration of $M$ whose subquotients are direct sums of standard modules. This means that it is enough to prove the claim in the case when $M$ is a direct sum of standard modules. In this case, the claim follows from Corollary 6(ii).

Proof of Theorem 14. Statement (i) follows from the additivity of the conditions that appear on the right-hand side in the formulae of Theorem 2.

The existence part of statement (ii) is proved using the usual approach of universal extensions (see [Ri]). We start with $\Delta(\lambda)$ and go down with respect to the preorder $\preceq$. If all first extensions from all (shifted) standard modules to $\Delta(\lambda)$ vanish, then $\Delta(\lambda) \in$ $\mathcal{F}^{\downarrow}(\bar{\nabla})$ by Theorem 2(ii). Otherwise there exist $\mu \in \Lambda$ and $j^{\prime} \in \mathbb{Z}$ such that

$$
\operatorname{ext}_{A}^{1}\left(\Delta(\mu)\left\langle j^{\prime}\right\rangle, \Delta(\lambda)\right) \neq 0 \text {. }
$$

We assume that $\mu$ is maximal with this property (recall that $\mu \prec \lambda$ by Lemma 16) and use Lemma 15 to choose $j^{\prime}$ such that

$$
\operatorname{ext}_{A}^{1}(\Delta(v)\langle j\rangle, \Delta(\lambda)) \neq 0
$$

implies that $j \leq j^{\prime}$ for all $\nu \in \bar{\mu}$.

For every $v \in \bar{\mu}$ and $j \leq j^{\prime}$, the space $\operatorname{ext}_{A}^{1}(\Delta(\nu)\langle j\rangle, \Delta(\lambda))$ is finite dimensional, say of dimension $l_{v, j}$. Consider the universal extension

$$
X \hookrightarrow Y \rightarrow Z,
$$

where $X=\Delta(\lambda)$ and

$$
Z=\bigoplus_{\nu \in \bar{\mu}} \bigoplus_{j \leq j^{\prime}} \Delta(\nu)\langle j\rangle^{l_{\nu, j}} \in \mathcal{F}^{\downarrow}(\Delta)
$$

(note that $\operatorname{ext}_{A}^{1}(Z, Z)=0$ by Lemma 16). By construction, $Y \in \mathcal{F}^{\downarrow}(\Delta)$. We further claim that $Y$ is indecomposable. Indeed, let $e \in \operatorname{end}_{A}(Y)$ be a nonzero idempotent 
(note that $e$ is homogeneous of degree zero). As $v \prec \lambda$, we have $\operatorname{hom}_{A}(\Delta(\lambda)$, $\Delta(v)\langle j\rangle)=0$ for any $v$ and $j$ as above. Therefore $e$ maps $X$ (which is indecomposable) to $X$. If $\left.e\right|_{X}=0$, then $e$ provides a splitting for a nontrivial direct summand of $Z$ in (21); if $\left.e\right|_{X}=\mathrm{id}_{X}$ and $e \neq \mathrm{id}_{Y}$, then $\operatorname{id}_{Y}-e \neq 0$ annihilates $X$ and hence provides a splitting for a nontrivial direct summand of $Z$ in (21). This contradicts our construction of $Y$ as the universal extension. Therefore $e=\operatorname{id}_{Y}$, which proves that the module $Y$ is indecomposable. By Lemma 16, there are no extensions between the summands of $Z$. Since $\operatorname{ext}_{A}^{1}(Z, Z)=0$ and our extension is universal,

$$
\operatorname{ext}_{A}^{1}(\Delta(v)\langle j\rangle, Y)=0
$$

for all $\nu \in \bar{\mu}$ and all $j$.

Now take the indecomposable module constructed in the previous paragraph as $X$, take a maximal $\mu^{\prime}$ such that $\operatorname{ext}_{A}^{1}\left(\Delta\left(\mu^{\prime}\right)\langle j\rangle, X\right) \neq 0$ for some $j$, and do the same thing as in the previous paragraph. Proceed inductively. In a finite number of steps, we end up with an indecomposable module $T(\lambda)$ such that $\Delta(\lambda) \hookrightarrow T(\lambda)$, the cokernel is in $\mathcal{F}^{\downarrow}(\Delta)$, and

$$
\operatorname{ext}_{A}^{1}(\Delta(\mu)\langle j\rangle, T(\lambda))=0
$$

for all $\mu$ and $j$. By Theorem 2(ii), we have $T(\lambda) \in \mathcal{F}^{\downarrow}(\bar{\nabla})$. This proves the existence part of statement (ii). The uniqueness part will follow from statement (iii).

Let $M \in \mathcal{F}^{\downarrow}(\Delta) \cap \mathcal{F}^{\downarrow}(\bar{\nabla})$ be indecomposable and $\Delta(\lambda) \hookrightarrow M$ be such that the cokernel Coker has a standard filtration. Applying $\operatorname{hom}_{A}(-, T(\lambda))$ to the short exact sequence

$$
\Delta(\lambda) \hookrightarrow M \rightarrow \text { Coker }
$$

we obtain the exact sequence

$$
\operatorname{hom}_{A}(M, T(\lambda)) \rightarrow \operatorname{hom}_{A}(\Delta(\lambda), T(\lambda)) \rightarrow \operatorname{ext}_{A}^{1}(\text { Coker, } T(\lambda)) .
$$

Here the right-hand term is zero by Lemma 17 and the definition of $T(\lambda)$. As the middle term is obviously nonzero, the left-hand term is nonzero as well. This gives us a nonzero map $\alpha$ from $M$ to $T(\lambda)$. Similarly, one constructs a nonzero map $\beta$ from $T(\lambda)$ to $M$ such that the composition $\alpha \circ \beta$ is the identity on $\Delta(\lambda)$.

LEMMA 18. Let $T(\lambda)$ be as above.

(i) For any $n \in \mathbb{Z}$, there exists a submodule $N^{(n)}$ of $T(\lambda)$ with the following properties:

(a) $N^{(n)}$ is indecomposable.

(b) $N^{(n)}$ has finite standard filtration starting with $\Delta(\lambda)$.

(c) $N_{i}^{(n)}=T(\lambda)_{i}$ for all $i \leq n$.

(d) Every endomorphism of $T(\lambda)$ restricts to an endomorphism of $N^{(n)}$.

(ii) The composition $\alpha \circ \beta$ is an automorphism of $T(\lambda)$.

Proof. Consider the multiset $\mathcal{M}$ of all standard subquotients of $T(\lambda)$. It might be infinite. However, for every $m \in \mathbb{Z}$, the multiset $\mathcal{M}_{m}$ of those subquotients $X$ of $T(\lambda)$ for which $X_{i} \neq 0$ for some $i \leq m$ is finite, since $T(\lambda) \in A^{\downarrow}$-mod. Construct the 
submultiset $\mathcal{N}$ of $\mathcal{M}$ in the following way. Start with $\mathcal{M}_{n} \cup\{\Delta(\lambda)\}$, which is finite. From Lemma 15 it follows that every subquotient from $\mathcal{M}_{n}$ has a nonzero first extension with finitely many other subquotients from $\mathcal{M}$. Add to $\mathcal{N}$ all such subquotients (counted with multiplicities); moreover, if we add some $\Delta(\mu)\langle j\rangle$, add as well all $\Delta(v)\langle i\rangle$, where $i \geq j$ and $\mu \preceq v$, that occur in $\mathcal{M}$. Obviously, the result will be a finite set. Now repeat the same procedure for all newly added subquotients and continue. By Lemma 16, at each successive step, we will add only those $\Delta(v)\langle i\rangle$ for which $\mu \prec v$ (this is a strict inequality!) for some minimal $\mu$ in the set indexing subquotients added on the previous step.

As $\Lambda$ is finite, after finitely many steps we will get a finite submultiset $\mathcal{N}$ of $\mathcal{M}$ with the following properties: no subquotient from $\mathcal{N}$ extends any subquotient from $\mathcal{M} \backslash \mathcal{N}$; there are no homomorphisms from any subquotient from $\mathcal{N}$ to any subquotient from $\mathcal{M} \backslash \mathcal{N}$. Using the vanishing of the first extension, one shows that there is a submodule $N^{(n)}$ of $T(\Lambda)$ that has a standard filtration such that the multiset of subquotients is precisely $\mathcal{N}$; in particular, $N^{(n)}$ satisfies (i)(b). By construction, $N^{(n)}$ also satisfies (i)(c). The vanishing of homomorphisms from subquotients from $\mathcal{N}$ to subquotients from $\mathcal{M} \backslash \mathcal{N}$ implies that $N^{(n)}$ satisfies (i)(d). That $N^{(n)}$ satisfies (i)(a) is proved similarly to the proof of the indecomposability of $T(\lambda)$. This proves statement (i).

To prove that $\alpha \circ \beta$ is an automorphism (statement (ii)) it is enough to show that for any $n \in \mathbb{Z}$ the restriction of $\alpha \circ \beta$ to $T(\lambda)_{n}$ is a linear automorphism. The restriction of $\alpha \circ \beta$ to $N^{(n)}$ (which is well-defined by (i)(d)) is not nilpotent as it is the identity on $\Delta(\lambda)$. As $A$ is positively graded, the space $\operatorname{hom}_{A}(\Delta(\mu), \Delta(\nu)\langle j\rangle)$ is finite dimensional for all $\mu, v$ and $j$. From this observation and (i)(b) it follows that the endomorphism algebra of $N^{(n)}$ is finite dimensional. This algebra is local by (i)(a). Therefore the restriction of $\alpha \circ \beta$ to $N^{(n)}$, being a nonnilpotent element of a local finite-dimensional algebra, is an automorphism. Therefore the restriction of $\alpha \circ \beta$ to all $N_{i}^{(n)}$, in particular, to $N_{n}^{(n)}=T(\lambda)_{n}$ (see (i)(c)), is a linear automorphism. This completes the proof.

After Lemma 18, substituting $\alpha$ by $(\alpha \circ \beta)^{-1} \circ \alpha$, we may assume that $\alpha \circ \beta=$ $\operatorname{id}_{T(\lambda)}$. Further, $\beta$ is injective and $\alpha$ is surjective. The gives us splittings for the following two short exact sequences:

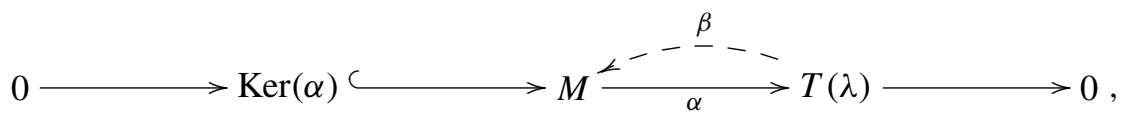

$$
\begin{aligned}
& 0 \longrightarrow T(\lambda) \stackrel{\left\llcorner-\frac{\alpha}{-}->\right.}{\longrightarrow} M \longrightarrow 0 \text { Coker }(\beta) \longrightarrow 0
\end{aligned}
$$

As $M$ is indecomposable by assumption, $\operatorname{Ker}(\alpha)=\operatorname{Coker}(\beta)=0$, which implies that $\alpha$ and $\beta$ are isomorphisms. Therefore $M \cong T(\lambda)$, which completes the proof of the theorem.

The objects of the category $\mathcal{F}^{\downarrow}(\Delta) \cap \mathcal{F}^{\downarrow}(\bar{\nabla})$ are called tilting modules. 
REMARK 19. Note that a tilting module may be an infinite direct sum of indecomposable tilting modules. Note also that the direct sum of all indecomposable tilting modules (with all shifts) does not belong to $A^{\downarrow}$-gmod. It might happen that it does not belong to $A$-gmod either, since local finiteness is an issue.

COROLLARY 20. Let A be a positively graded standardly stratified algebra.

(i) Every $M \in \mathcal{F}^{\downarrow}(\Delta)$ has a coresolution by tilting modules of length at most $|\Lambda|-1$.

(ii) Every $M \in \mathcal{F}^{\downarrow}(\bar{\nabla})$ has a (possibly infinite) resolution by tilting modules.

PROOF. This follows from Theorem 14 and the definitions by standard arguments.

REMARK 21. Note that the standard filtration of $T(\lambda)$ may be infinite, see Example 43.

Unfortunately, Remark 21 says that one cannot hope for a reasonable analogue of Ringel duality on the class of algebras we consider. We can of course consider the endomorphism algebra of the direct sum of all tilting modules, but from Remark 21 it follows that projective modules over such algebras might have infinite standard filtrations and hence we will not be able to construct tilting modules for them. Another obstruction is that we actually cannot guarantee that the induced grading on this endomorphism algebra will be positive (see examples in [Ma, MO]). To deal with these problems we have to introduce some additional restrictions.

\section{Ringel duality for graded standardly stratified algebras}

Consider the $\mathbb{k}$-linear category $\mathfrak{T}$, which is the full subcategory of $A^{\downarrow}$-gmod, whose objects are the $T(\lambda)\langle j\rangle$, where $\lambda \in \Lambda$ and $j \in \mathbb{Z}$. The group $\mathbb{Z}$ acts freely on $\mathfrak{T}$ via $\langle j\rangle$ and the quotient of $\mathfrak{T}$ by this free action is a $\mathbb{Z}$-graded $\mathbb{k}$-linear category $\overline{\mathfrak{T}}$, whose objects can be identified with $T(\lambda)$, where $\lambda \in \Lambda$ (see [DM, MOS] for more details). Thus the ungraded endomorphism algebra $R(A)=\operatorname{End}_{A}(T)$, where $T=\bigoplus_{\lambda \in \Lambda} T(\lambda)$, becomes a $\mathbb{Z}$-graded $\mathbb{k}$-algebra in the natural way. The algebra $R(A)$ is called the Ringel dual of $A$. The algebra $A$ will be called weakly adapted provided that every $T(\lambda)$, where $\lambda \in \Lambda$, has a finite standard filtration. The algebra $A$ will be called adapted provided that the above $\mathbb{Z}$-grading on $R(A)$ is positive.

PROPOSITION 22. The following hold.

(i) Any adapted algebra is weakly adapted.

(ii) If $A$ is weakly adapted, then $R(A)$ is locally finite.

PROOF. Because of Lemma 5 and the definition of tilting modules, every homomorphism from $T(\lambda)$ to $T(\mu)\langle j\rangle$ is induced from a homomorphism from some standard subquotient of $T(\lambda)$ to some proper standard subquotient of $T(\mu)\langle j\rangle$.

Since $\bar{\nabla}(\mu)\langle j\rangle$ is a (sub)quotient of $T(\mu)\langle j\rangle$, the condition that the above $\mathbb{Z}$-grading on $R(A)$ is positive implies that every standard subquotient of $T(\lambda)$, 
different from $\Delta(\lambda)$, must have the form $\Delta(\mu)\langle j\rangle$ for some $j>0$. However, the vector space $\bigoplus_{j \leq 0} T(\lambda)_{j}$ is finite dimensional as $T(\lambda) \in A^{\downarrow}$-gmod, which shows that any standard filtration of $T(\lambda)$ must be finite. This proves statement (i).

Statement (ii) follows from the finiteness of a standard filtration of $T(\lambda)$ and the obvious fact that $\operatorname{hom}_{A}(\Delta(\lambda), M)$ is finite dimensional for any $M \in A$-gmod.

Corollary 23. Assume that $A$ is adapted. Then every $M \in \mathcal{F}^{b}(\Delta)$, in particular, every indecomposable projective A-module, has a finite coresolution,

$$
0 \rightarrow M \rightarrow T_{0} \rightarrow T_{1} \rightarrow \cdots \rightarrow T_{k} \rightarrow 0
$$

such that every $T_{i}$ is a finite direct sum of indecomposable tilting A-modules.

PROOF. It is enough to prove the result when $M=\Delta(\lambda)$. It is obvious when $\lambda$ is minimal, as $\Delta(\lambda)=T(\lambda)$ in this case. From Theorem 14(ii), we have the exact sequence

$$
0 \rightarrow \Delta(\lambda) \rightarrow T(\lambda) \rightarrow \text { Coker }
$$

and Coker has a standard filtration with possible subquotients $\Delta(\mu)\langle i\rangle$, where $\mu \prec \lambda$ and $i \in \mathbb{Z}$. By Proposition 22(i), the standard filtration of Coker is finite and hence the claim follows by induction (with respect to the partial preorder $\preceq$ ).

A complex $\mathcal{X}^{\bullet}$ of $A$-modules is called perfect provided that it is bounded and every nonzero $\mathcal{X}^{i}$ is a direct sum of finitely many indecomposable modules. Let $\mathcal{P}(A)$ denote the homotopy category of perfect complexes of graded projective $A$-modules. As every indecomposable projective $A$-module has a finite standard filtration, it follows by induction that $\mathcal{F}^{b}(\Delta) \subseteq \mathcal{P}(A)$. Consider the contravariant functor

$$
\mathrm{G}=\mathcal{R} \operatorname{hom}_{A}(-, \mathfrak{T})
$$

(see [MOS] for details of hom-functors for $\mathbb{k}$-linear categories). As we will see in Theorem 24(iii), the functor $\mathrm{G}$ is a functor from $\mathcal{P}(A)$ to $\mathcal{P}(R(A))$. To distinguish $A$ and $R(A)$-modules, if necessary, we will use $A$ and $R(A)$ as superscripts for the corresponding modules.

THEOREM 24 (Weak Ringel duality). Let A be an adapted standardly stratified algebra.

(i) The algebra $R(A)$ is an adapted standardly stratified algebra with respect to $\preceq$ op.

(ii) We have $R(R(A)) \cong A$.

(iii) The functor $\mathrm{G}$ is an antiequivalence from $\mathcal{P}(A)$ to $\mathcal{P}(R(A))$.

(iv) The functor $\mathrm{G}$ induces an antiequivalence between $\mathcal{F}^{b}\left(\Delta^{(A)}\right)$ and $\mathcal{F}^{b}\left(\Delta^{(R(A))}\right)$, which sends standard A-modules to standard $R(A)$-modules, tilting A-modules to projective $R(A)$-modules and projective A-modules to tilting $R(A)$-modules. 
ProOf. By construction, the functor $\mathrm{G}$ maps indecomposable tilting $A$-modules to indecomposable projective $R(A)$-modules. From Corollary 23 it follows that every indecomposable projective $A$-module $M$ has a coresolution of the form (22), such that every $T_{i}$ is a finite direct sum of indecomposable tilting $A$-modules. This implies that every object in $\mathcal{P}(A)$ can be represented by a perfect complex of tilting modules. This shows that $\mathrm{G}$ maps $\mathcal{P}(A)$ to $\mathcal{P}(R(A))$. As $T$ is a tilting module, statement (iii) follows directly from the Rickard-Morita theorem for $\mathbb{k}$-linear categories; see, for instance, [Ke, Corollary 9.2] or [DM, Theorem 2.1].

The functor $\mathrm{G}$ is acyclic, and, in particular, it is exact on $\mathcal{F}^{b}\left(\Delta^{(A)}\right)$ by Lemma 5 . By construction, it maps tilting $A$-modules to projective $R(A)$-modules and thus projective $R(A)$-modules have filtrations by images (under $\mathrm{G}$ ) of standard $A$-modules. By Proposition 22, these filtrations of projective $R(A)$-modules by images of standard $A$-modules are finite. As in the classical case (see [Ri]), it is easy to see that the images of standard $A$-modules are standard $R(A)$-modules (with respect to $\preceq^{\mathrm{op}}$ ). From Proposition 22(ii) and our assumptions, it follows that the algebra $R(A)$ is positively graded. This implies that $R(A)$ is a graded standardly stratified algebra (with respect to $\preceq$ op).

Because of our description of standard modules for $R(A)$, the functor $\mathrm{G}$ maps $\mathcal{F}^{b}\left(\Delta^{(A)}\right)$ to $\mathcal{F}^{b}\left(\Delta^{(R(A))}\right)$. In particular, projective $A$-modules are also mapped to some modules in $\mathcal{F}^{b}\left(\Delta^{(R(A))}\right)$. Since $\mathrm{G}$ is a derived equivalence by (iii), for $i>0$, $j \in \mathbb{Z}$ and $\lambda, \mu \in \Lambda$, we see that

$$
\operatorname{ext}_{R(A)}^{i}(\mathrm{G} \Delta(\lambda)\langle j\rangle, \mathrm{G} P(\mu))=\operatorname{ext}_{A}^{i}(P(\mu), \Delta(\lambda)\langle j\rangle)=0 .
$$

Hence $\mathrm{G} P(\mu)$ has a proper costandard filtration by Theorem 2(i), and thus is a tilting $R(A)$-module, which implies statement (ii). As projective $A$-modules have finite standard filtration, the algebra $R(A)$ is weakly adapted. It is even adapted as the grading on $R(R(A))$ coincides with the grading on $A$ and is hence positive. This proves statement (i). Statement (iv) follows easily from the properties of G, established above. This completes the proof.

Similarly, we consider the contravariant functors

$$
\begin{aligned}
\mathrm{F} & =\mathcal{R} \operatorname{hom}_{A}\left(\mathfrak{T},{ }_{-}\right)^{\circledast}: \mathcal{D}^{+}\left(A^{\uparrow} \text {-gmod }\right) \rightarrow \mathcal{D}^{-}\left(R(A)^{\downarrow} \text {-gmod }\right), \\
\tilde{\mathrm{F}} & =\mathcal{R} \operatorname{hom}_{A}\left(\mathfrak{T},{ }_{-}\right)^{\circledast}: \mathcal{D}^{-}\left(A^{\downarrow} \text {-gmod }\right) \rightarrow \mathcal{D}^{+}\left(R(A)^{\uparrow} \text {-gmod }\right) .
\end{aligned}
$$

Although it is not obvious at first glance, the following statement bears a strong resemblance to [MOS, Proposition 20].

THEOREM 25 (Strong Ringel duality). Let $A$ be an adapted standardly stratified algebra.

(i) Both $\mathrm{F}$ and $\tilde{\mathrm{F}}$ are antiequivalences.

(ii) The functor $\mathrm{F}$ induces an antiequivalence from the category $\mathcal{F}^{\uparrow}\left(\bar{\nabla}^{(A)}\right)$ to the category $\mathcal{F}^{\downarrow}\left(\bar{\nabla}^{(R(A))}\right)$ that sends proper costandard A-modules to proper costandard $R(A)$-modules, and injective A-modules to tilting $R(A)$-modules. 
(iii) The functor $\tilde{\mathrm{F}}$ induces an antiequivalence from the category $\mathcal{F}^{\downarrow}\left(\bar{\nabla}^{(A)}\right)$ to the category $\mathcal{F}^{\uparrow}\left(\bar{\nabla}^{(R(A))}\right)$ that sends proper costandard A-modules to proper costandard $R(A)$-modules, and tilting A-modules to injective $R(A)$-modules.

PROOF. Consider the covariant versions of our functors:

$$
\begin{aligned}
\mathrm{H} & =\mathcal{R} \operatorname{hom}_{A}\left(\mathfrak{T},{ }_{-}\right): \mathcal{D}^{+}\left(A^{\uparrow} \text {-gmod }\right) \rightarrow \mathcal{D}^{+}\left(\operatorname{gmod}-R(A)^{\uparrow}\right), \\
\tilde{\mathrm{H}} & =\mathcal{R} \operatorname{hom}_{A}\left(\mathfrak{T},{ }_{-}\right): \mathcal{D}^{-}\left(A^{\downarrow}-\operatorname{gmod}\right) \rightarrow \mathcal{D}^{-}\left(\operatorname{gmod}-R(A)^{\downarrow}\right) .
\end{aligned}
$$

Every object in $\mathcal{D}^{-}\left(A^{\downarrow}\right.$-gmod) has a projective resolution. Since $T$ is a tilting module, every object in $\mathcal{D}^{-}\left(A^{\downarrow}\right.$-gmod) is also given by a complex of tilting modules. As tilting modules are self-orthogonal, for complexes of tilting modules, the functor $\tilde{\mathrm{H}}$ reduces to the usual hom functor. Similarly every object in $\mathcal{D}^{+}\left(A^{\uparrow}\right.$-gmod) has an injective resolution, and for such complexes, the functor $\mathrm{H}$ reduces to the usual hom functor.

The left adjoints $\mathrm{H}^{\prime}$ and $\tilde{\mathrm{H}}^{\prime}$ of $\mathrm{H}$ and $\tilde{\mathrm{H}}$ are thus given by the left derived functors of the tensoring with $\mathfrak{T}$. As $T$ is a tilting module, these left adjoint functors can be given as a tensoring with a finite tilting complex of $A-R(A)$-bimodules, projective as right $R(A)$-modules, followed by taking the total complex.

Using the definition of proper costandard modules it is straightforward to verify that both $\mathrm{H}$ and $\tilde{\mathrm{H}}$ map proper costandard left $A$-modules to proper standard right $R(A)$-modules. Similarly, both $\mathrm{H}^{\prime}$ and $\tilde{\mathrm{H}}^{\prime}$ map proper standard right $R(A)$-modules to proper costandard left $A$-modules. Since proper (co)standard objects have trivial endomorphism rings, it follows by standard arguments that the adjunction morphisms

$$
\begin{aligned}
\operatorname{Id}_{\mathcal{D}^{+}\left(\operatorname{gmod}-R(A)^{\uparrow}\right)} \rightarrow \mathrm{HH}^{\prime}, & \mathrm{H}^{\prime} \mathrm{H} \rightarrow \mathrm{Id}_{\mathcal{D}^{+}\left(A^{\uparrow} \text {-gmod }\right)}, \\
\operatorname{Id}_{\mathcal{D}^{-}\left(\operatorname{gmod}-R(A)^{\downarrow}\right)} \rightarrow \tilde{\mathrm{H}} \tilde{H}^{\prime}, & \tilde{H}^{\prime} \tilde{\mathrm{H}} \rightarrow \mathrm{Id}_{\mathcal{D}^{-}\left(A^{\downarrow} \text {-gmod }\right)}
\end{aligned}
$$

induce isomorphisms, when evaluated on the appropriate proper (co)standard objects. Therefore the adjunction morphisms above are isomorphisms of functors on the categories that are generated (as triangular categories) by proper (co)standard objects. Using the classical limit construction (see [Ric]), one shows that both $\mathrm{H}$ and $\tilde{\mathrm{H}}$ are equivalences of categories. This shows that both $\mathrm{F}$ and $\tilde{\mathrm{F}}$ are antiequivalences of categories. This proves statement (i), and statements (ii) and (iii) easily follow.

\section{Proof of the main result}

When $M \in\{P(\lambda), I(\lambda), T(\lambda), \Delta(\lambda), \bar{\nabla}(\lambda)\}$, we will say that the centroid of the graded module $M\langle j\rangle$, where $j \in \mathbb{Z}$, belongs to $-j$. Let $\mathcal{X}^{\bullet}$ and $\mathcal{Y}^{\bullet}$ be two complexes of tilting modules, both bounded from the right. A complex $\mathcal{X}^{\bullet}$ of projective, injective, tilting, standard, or costandard modules is called linear provided that the centroids of all indecomposable summands of $\mathcal{X}^{i}$ belong to $-i$ for all $i$. A positively graded algebra $B$ is called Koszul if all simple $B$-modules have linear projective resolutions. The Koszul dual $E(A)$ of a Koszul algebra $A$ is just the Yoneda extension algebra of the direct sum of all simple $A$-modules. The algebra $E(A)$ is positively graded by the degree of extensions. 
We will say that $\mathcal{X}^{\bullet}$ dominates $\mathcal{Y}^{\bullet}$ provided that the following holds for all $i \in \mathbb{Z}$ : if the centroid of an indecomposable summand of $\mathcal{X}^{i}$ belongs to $j$ and the centroid of an indecomposable summand of $\mathcal{Y}^{i}$ belongs to $j^{\prime}$, then $j<j^{\prime}$.

The aim of this section is to prove Theorem 1. For this, we fix throughout an algebra $A$ satisfying the assumptions of Theorem 1 (we will call such algebra balanced). For $\lambda \in \Lambda$, we denote by $\mathcal{S}_{\lambda}^{\bullet}$ and $\mathcal{C}_{\lambda}^{\bullet}$ the linear tilting coresolution of $\Delta(\lambda)$ and resolution of $\bar{\nabla}(\lambda)$, respectively. We will proceed along the lines of [Ma, Section 3] and do not repeat the arguments, which are similar to those of [Ma, Section 3].

LEMMA 26. The algebra $A$ is adapted.

ProOF. Mutatis mutandis, this is [Ma, Lemma 2].

Corollary 27. We have $\operatorname{hom}_{A}(T(\lambda)\langle i\rangle, T(\mu))=0$, for all $\lambda, \mu \in \Lambda$ and $i \in \mathbb{N}$.

Corollary 28. Let $\mathcal{X}^{\bullet}$ and $\mathcal{Y}^{\bullet}$ be two complexes of tilting modules, both bounded from the right. Assume that $\mathcal{X}^{\bullet}$ dominates $\mathcal{Y}^{\bullet}$. Then $\operatorname{Hom}_{\mathcal{D}^{-}(A)}\left(\mathcal{X}^{\bullet}, \mathcal{Y}^{\bullet}\right)=0$.

PROOF. Mutatis mutandis, this is [Ma, Corollary 4].

Proposition 29. For every $\lambda \in \Lambda$, the module $L(\lambda)$ is isomorphic in $\mathcal{D}^{-}(A)$ to a linear complex $\mathcal{L}_{\lambda}^{\bullet}$ of tilting modules.

Proof. Just as in [Ma, Proposition 5], one constructs a complex $\overline{\mathcal{P}}^{\bullet}$ of tilting modules in $\mathcal{D}^{-}(A)$, quasi-isomorphic to $L(\lambda)$, and such that for each $i$ all centroids of indecomposable summands in $\overline{\mathcal{P}}^{i}$ belong to some $j$ no greater than $-i$.

Let us now prove the claim by induction with respect to $\preceq$. If $\lambda$ is minimal, then $L(\lambda)=\bar{\nabla}(\lambda)$, and we can take $\mathcal{L}_{\lambda}^{\bullet}=\mathcal{C}_{\lambda}^{\bullet}$. Otherwise, consider the short exact sequence

$$
0 \rightarrow L(\lambda) \rightarrow \bar{\nabla}(\lambda) \rightarrow \text { Coker } \rightarrow 0 .
$$

Since $A$ is positively graded, $\operatorname{Coker}_{j}=0$ for all $j \geq 0$. Moreover, Coker is finite dimensional (by Lemma 4) and all simple subquotients of Coker correspond to some $\mu \in \Lambda$ such that $\mu \prec \lambda$. Using the inductive assumption, we can resolve every simple subquotient of Coker using the corresponding linear complexes of tilting modules and thus deduce that Coker is quasi-isomorphic to some complex $\mathcal{X}^{\bullet}$ of tilting modules such that for each $i$ all centroids of indecomposable summands in $\mathcal{X}^{i}$ belong to some $j$ no greater than $-i-1$. As $\bar{\nabla}(\lambda)$ has a linear tilting resolution, it follows that $L(\lambda)$ is quasi-isomorphic to some complex $\bar{Q}^{\bullet}$ of tilting modules, such that for each $i$ all centroids of indecomposable summands in $\overline{\mathcal{Q}}^{i}$ belong to some $j$ no greater than $-i$.

Because of the uniqueness of the minimal tilting complex $\mathcal{L}_{\lambda}^{\bullet}$ representing $L(\lambda)$ in $\mathcal{D}^{-}\left(A^{\downarrow}\right.$-mod), we conclude that for all $i \in \mathbb{Z}$ the centroids of all indecomposable summands in $\mathcal{L}_{\lambda}^{i}$ belong to $-i$. This means that $\mathcal{L}_{\lambda}^{\bullet}$ is linear and completes the proof.

Corollary 30. The algebra $A$ is Koszul.

PROOF. Mutatis mutandis, this is [Ma, Corollary 6]. 
COROLlary 31. The following hold.

(i) Standard A-modules have linear projective resolutions.

(ii) Proper costandard A-modules have linear injective coresolutions.

Proof. Assume that $\operatorname{ext}_{A}^{i}(\Delta(\lambda), L(\mu)\langle j\rangle) \neq 0$ for some $\lambda, \mu \in \Lambda, i \geq 0$ and $j \in \mathbb{Z}$. As $A$ is positively graded we obviously have $j \leq-i$. On the other hand, this inequality yields the existence of a nonzero homomorphism (in $\mathcal{D}^{-}\left(A^{\downarrow}\right.$-mod)) from $\mathcal{S}_{\lambda}^{\bullet}$ to $\mathcal{L}_{\lambda}^{\bullet}[i]\langle j\rangle$. However, both $\mathcal{S}_{\lambda}^{\bullet}$ and $\mathcal{L}_{\lambda}^{\bullet}$ are linear by Proposition 29 , and hence from Corollary 28 it follows that $j \geq-i$. Therefore $j=-i$ and statement (i) follows. The statement (ii) is proved similarly.

COROLlary 32. The following hold.

(i) Standard $R(A)$-modules have finite linear projective resolutions.

(ii) Standard $R(A)$-modules have finite linear tilting coresolutions.

(iii) Proper costandard $R(A)$-modules have linear tilting resolutions.

(iv) Proper costandard $R(A)$-modules have linear injective coresolutions.

Proof. Using Theorem 24(iv), we see that the functor $G$ maps a finite linear projective resolution of $\Delta^{(A)}$ (found in Corollary 31(i)) to a finite linear tilting coresolution of $\Delta^{(R(A))}$. It also maps a finite linear tilting coresolution of $\Delta^{(A)}$ to a finite linear projective resolution of $\Delta^{(R(A))}$.

Using Theorem 25(ii) we see that the functor $F$ maps a linear injective coresolution of $\bar{\nabla}^{(A)}$ (found in Corollary 31(ii)) to a linear tilting resolution of $\bar{\nabla}^{(R(A))}$. Using Theorem 25(iii) we see that the functor $\tilde{\mathrm{F}}$ maps a linear tilting resolution of $\bar{\nabla}^{(A)}$ to a linear injective coresolution of $\bar{\nabla}^{(R(A))}$. The corollary follows.

Corollary 33. The algebra $R(A)$ is Koszul.

PROOF. This follows from Corollaries 30 and 32.

Denote by $\mathfrak{L} \mathfrak{T}$ the full subcategory of $\mathcal{D}^{-}(A)$ that consists of all linear complexes of tilting $A$-modules. The category $\mathfrak{L} \mathfrak{T}$ is equivalent to gmod- $E(R(A))^{\uparrow}$ and simple objects of $\mathfrak{L} \mathfrak{T}$ have the form $T(\lambda)\langle-i\rangle[i]$, where $\lambda \in \Lambda$ and $i \in \mathbb{Z}$ (see [MOS]).

PROPOSITION 34. The following hold.

(i) The objects $\mathcal{S}_{\lambda}^{\bullet}$, where $\lambda \in \Lambda$, are proper standard objects in $\mathfrak{L} \mathfrak{T}$ with respect to $\preceq$.

(ii) The objects $\mathcal{C}_{\lambda}^{\bullet}$, where $\lambda \in \Lambda$, are costandard objects in $\mathfrak{L} \mathfrak{T}$ with respect to $\preceq$.

PROOF. Mutatis mutandis, this is [Ma, Proposition 11].

Proposition 35. For all $\lambda, \mu \in \Lambda$ and $i, j \in \mathbb{Z}$,

$$
\operatorname{Hom}_{\mathcal{D}^{b}(\mathfrak{L T})}\left(\mathcal{S}_{\lambda}^{\bullet}, \mathcal{C}_{\mu}\langle j\rangle[-i]^{\bullet}\right)= \begin{cases}\mathbb{k} & \text { if } \lambda=\mu \text { and } i=j=0 \\ 0 & \text { otherwise }\end{cases}
$$


PROOF. Mutatis mutandis, this is [Ma, Proposition 12].

COROLlARY 36. The algebra $E(R(A))$ is standardly stratified with respect to $\preceq$.

PROOF. Applying the duality to Propositions 34 and 35, we see that standard $E(R(A))$-modules are left orthogonal to proper costandard modules. Using this fact and the same arguments as in the proof of Theorem 2, one shows that projective $E(R(A))$-modules have a standard filtration.

Since standard $E(R(A))$-modules are left orthogonal to proper costandard modules, to prove that the standard filtration of an indecomposable projective $E(R(A))$-module is finite, it is enough to show that the dimension of the full ungraded homomorphism space from any indecomposable projective $E(R(A))$-module to any proper costandard module is finite. In terms of the category $\mathfrak{L T}$ (which gives the dual picture), we thus have to show that the dimension $N$ of the full ungraded homomorphism space from $\mathcal{S}_{\lambda}^{\bullet}$ to any injective object in $\mathfrak{L} \mathfrak{T}$ is finite. Realizing $\mathfrak{L} \mathfrak{T}$ as linear complexes of projective $R(A)$-modules, we know that injective objects of $\mathfrak{L} \mathfrak{T}$ are linear projective resolutions of simple $R(A)$-modules (see [MOS, Proposition 11]), while the proper standard objects are linear projective resolutions of standard $R(A)$-modules. We thus get that $N$ is bounded by the sum of the dimensions of all extension from the corresponding standard module to the corresponding simple module. Now the claim follows from the fact that all standard $R(A)$-modules have finite linear resolutions, by Corollary 32(i).

COROLlary 37. The complexes $\mathcal{L}_{\lambda}^{\bullet}$, where $\lambda \in \Lambda$, are tilting objects in $\mathfrak{L} \mathfrak{T}$.

PROOF. Mutatis mutandis, this is [Ma, Corollary 14].

COROLLARY 38. There is an isomorphism $E(A) \cong R(E(R(A)))$ of graded algebras, both considered with respect to the natural grading induced from $\mathcal{D}^{-}(A)$. In particular, $R(E(A)) \cong E(R(A))$.

PROOF. Mutatis mutandis, this is [Ma, Corollary 15].

COROLlary 39. Both $E(A)$ and $R(E(A))$ are positively graded with respect to the natural grading induced from $\mathcal{D}^{-}(A)$.

PROOF. Mutatis mutandis, this is [Ma, Corollary 16].

LEMMA 40. The algebra $E(R(A))$ is standard Koszul.

ProOF. Mutatis mutandis, this is [Ma, Lemma 18].

PROpOSITION 41. The positively graded algebras $E(A)$ and $R(E(A))$ are balanced.

ProOF. Mutatis mutandis, this is [Ma, Proposition 17]. 
Proof of Theorem 1. Statement (i) follows from Corollaries 30 and 31. Statement (ii) follows from Corollary 32 and Proposition 41. Statement (iii) follows from Proposition 29. Finally, statement (iv) follows from Corollary 38.

\section{Examples}

EXAMPLE 42. Consider the path algebra $A$ of the following quiver:

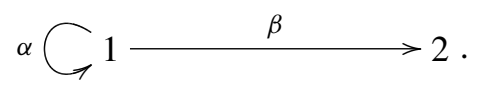

It is positively graded in the natural way (each arrow has degree one). We have $\Delta(2)=P(2)=L(2)$, while the projective module $P(1)$ looks as follows.

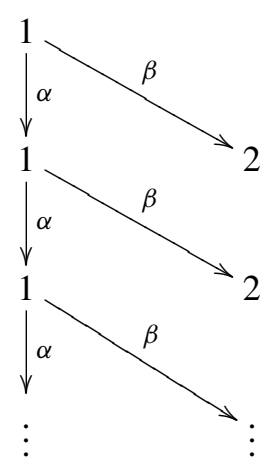

In particular, the ungraded composition multiplicity of $L(2)$ in $P(1)$ is infinite and hence $P(1)$ has an infinite standard filtration. In particular, Lemma 15 fails in this case, and hence the universal extension procedure does not have a starting point and cannot give us a module from $A^{\downarrow}$-gmod.

EXAMPLE 43. Consider the path algebra $B$ of the following quiver:

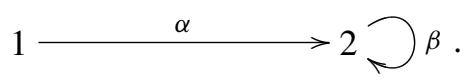

It is positively graded in the natural way (each arrow has degree one). We have $\Delta(1)=L(1)=T(1), \Delta(2)=P(2)$ and the following projective $B$-modules.

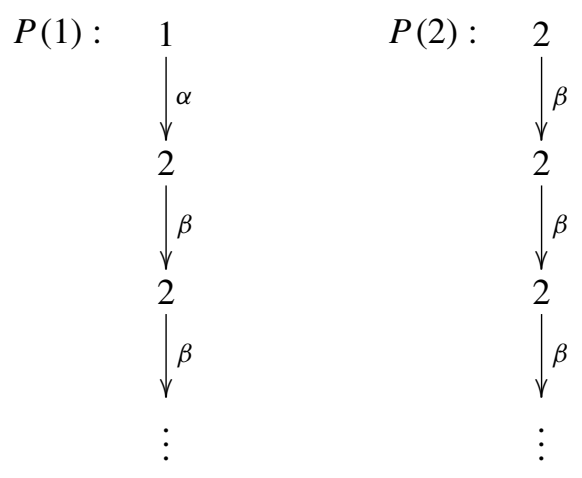


The module $T(2)$ looks as follows.

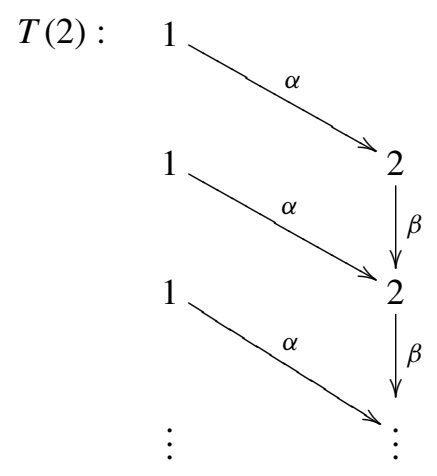

In particular, $T(2)$ has an infinite standard filtration and hence the algebra $B$ is not weakly adapted.

EXAMPLE 44. Consider the path algebra $C$ of the following quiver:

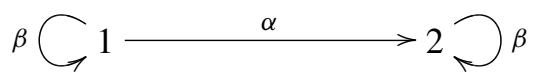

modulo the ideal generated by the relation $\alpha \beta=\beta \alpha$. It is positively graded in the natural way (each arrow has degree one). We have $\bar{\nabla}(1)=L(1)$ and also the following projective, standard, proper costandard and tilting $C$-modules.
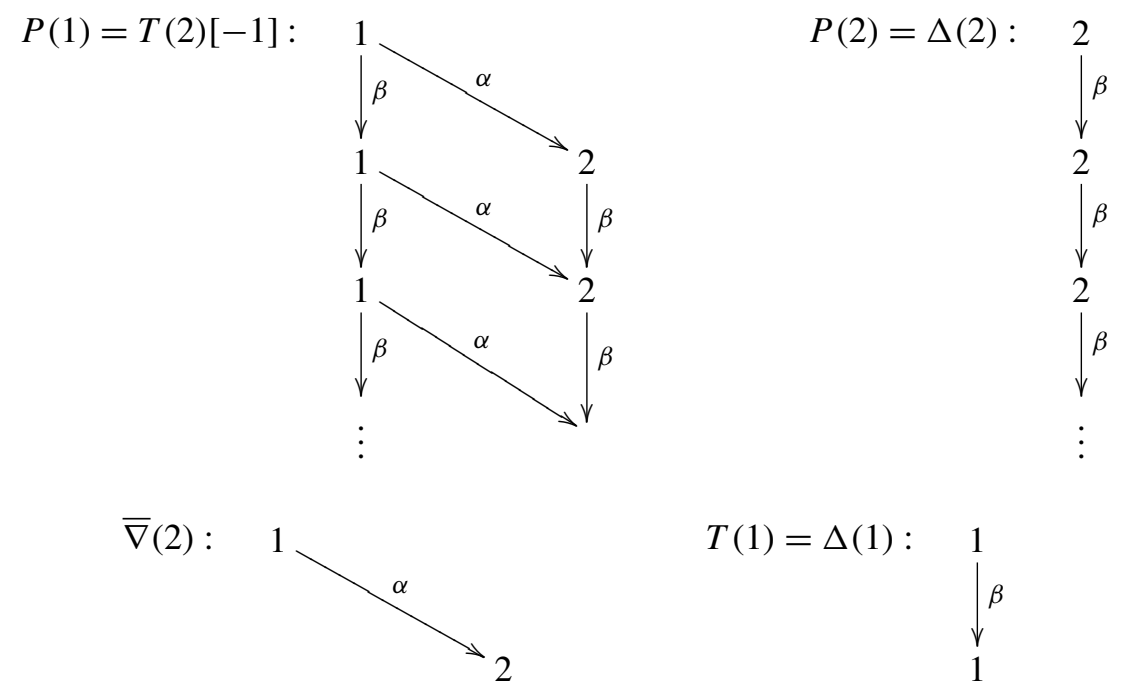
Standard and proper costandard $C$-modules have the following linear tilting (co)resolutions:

$$
\begin{gathered}
0 \rightarrow \Delta(1) \rightarrow T(1) \rightarrow 0, \\
0 \rightarrow \Delta(2) \rightarrow T(2) \rightarrow T(1)[1] \rightarrow 0, \\
0 \rightarrow T(1)[-1] \rightarrow T(1) \rightarrow \bar{\nabla}(1) \rightarrow 0 \\
0 \rightarrow T(2)[-1] \rightarrow T(2) \rightarrow \bar{\nabla}(2) \rightarrow 0 .
\end{gathered}
$$

Hence $C$ is balanced. The indecomposable tilting objects in $\mathfrak{L} \mathfrak{T}$ are given by

$$
\begin{gathered}
0 \rightarrow T(1)[-1] \rightarrow T(1) \rightarrow 0 \\
0 \rightarrow T(2)[-1] \rightarrow T(2) \oplus T(1) \rightarrow T(1)[1] \rightarrow 0 .
\end{gathered}
$$

We have $R(C) \cong C^{\text {op }}$, and $E(C)$ is the path algebra of the quiver:

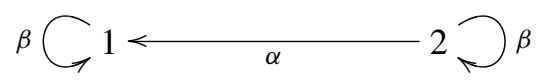

modulo the ideal generated by the relation $\alpha \beta=\beta \alpha$ and $\beta^{2}=0$, and furthermore $R(E(C)) \cong E(R(C)) \cong E(C)^{\mathrm{op}}$.

EXAMPLE 45. Every Koszul positively graded local algebra $A$ with $\operatorname{dim}_{\mathbb{k}} A_{0}=1$ is balanced. Every Koszul positively graded algebra is balanced in the case when $\prec$ is the full relation.

EXAMPLE 46. It follows directly from the definition that if the algebra $A$ is balanced, then the algebra $A / A e_{\bar{\lambda}} A$ is also balanced, for any maximal $\lambda$. It is also easy to see that if $A$ and $B$ are balanced, then both $A \oplus B$ and $A \otimes_{\mathbb{k}} B$ are balanced.

\section{Acknowledgements}

The research was partly supported by the Swedish Research Council. Some of the results in the paper were obtained during the author's visit to the Department of Algebra and Number Theory, Eőtvős University, Budapest in September 2008. The hospitality of Eôtvős University is gratefully acknowledged. The author also thanks István Ágoston and Erzsébet Lukács for their hospitality and many stimulating discussions, and is grateful to the referee for the very careful reading of the paper, for pointing out several inaccuracies and a gap in the original version, and for numerous suggestions which led to improvements in the original version of the paper.

\section{References}

[ADL1] I. Ágoston, V. Dlab and E. Lukács, 'Quasi-hereditary extension algebras', Algebr. Represent. Theory 6(1) (2003), 97-117.

[ADL2] I. Ágoston, V. Dlab and E. Lukács, 'Standardly stratified extension algebras', Comm. Algebra 33(5) (2005), 1357-1368. 
[AHLU] I. Ágoston, D. Happel, E. Lukács and L. Unger, 'Standardly stratified algebras and tilting', J. Algebra 226(1) (2000), 144-160.

[AB] M. Auslander and R.-O. Buchweitz, 'The homological theory of maximal Cohen-Macaulay approximations', in: Colloque en l'honneur de Pierre Samuel (Orsay, 1987), Mém. Soc. Math. Fr. (N.S.), 38 (1989), 5-37.

[AR] M. Auslander and I. Reiten, 'Applications of contravariantly finite subcategories', Adv. Math. 86(1) (1991), 111-152.

[CT] J. Chuang and W. Turner, 'Cubist algebras', Adv. Math. 217(4) (2008), 1614-1670.

[CPS2] E. Cline, B. Parshall and L. Scott, 'The homological dual of a highest weight category', Proc. Lond. Math. Soc. (3) 68(2) (1994), 294-316.

[CPS1] E. Cline, B. Parshall and L. Scott, 'Stratifying endomorphism algebras', Mem. Amer. Math. Soc. 124(591) (1996).

[D1] V. Dlab, 'Quasi-hereditary algebras revisited', in Representation Theory of Groups, Algebras, and Orders (Constanta, 1995), An. Ştiinţ. Univ. 'Ovidius' Constanţa Ser. Mat. 4(2) (1996), 43-54.

[DM] Y. Drozd and V. Mazorchuk, 'Koszul duality for extension algebras of standard modules', J. Pure Appl. Algebra 211(2) (2007), 484-496.

[Fr1] A. Frisk, 'Two-step tilting for standardly stratified algebras', Algebra Discrete Math. 3(3) (2004), 38-59.

[Fr2] A. Frisk, 'Dlab's theorem and tilting modules for stratified algebras', J. Algebra 314(2) (2007), 507-537.

[Fr3] A. Frisk, 'Typical blocks of the category $\mathcal{O}$ for the queer Lie superalgebra', J. Algebra Appl. 6(5) (2007), 731-778.

[Ke] B. Keller, 'Deriving DG categories', Ann. Sci. École Norm. Sup. (4) 27(1) (1994), 63-102.

[KKM] O. Khomenko, S. Koenig and V. Mazorchuk, 'Finitistic dimension and tilting modules for stratified algebras', J. Algebra 286(2) (2005), 456-475.

[Ma] V. Mazorchuk, 'Koszul duality for stratified algebras I. Balanced quasi-hereditary algebras', Manuscripta Math. 131 (2010), 1-10.

[MO] V. Mazorchuk and S. Ovsienko, 'A pairing in homology and the category of linear complexes of tilting modules for a quasi-hereditary algebra', J. Math. Kyoto Univ. 45(4) (2005), 711-741; With an appendix by C. Stroppel.

[MOS] V. Mazorchuk, S. Ovsienko and C. Stroppel, 'Quadratic duals, Koszul dual functors, and applications', Trans. Amer. Math. Soc. 361 (2009), 1129-1172.

[MT] V. Miemietz and W. Turner, 'Rational representations of $\mathrm{GL}_{2}$ ', Glasg. Math. J., to appear.

[Ric] J. Rickard, 'Derived equivalences as derived functors', J. Lond. Math. Soc. (2) 43(1) (1991), 37-48.

[Ri] C. Ringel, 'The category of modules with good filtrations over a quasi-hereditary algebra has almost split sequences', Math. Z. 208(2) (1991), 209-223.

\section{VOLODYMYR MAZORCHUK, Department of Mathematics, Uppsala University,}

SE 471 06, Uppsala, Sweden

e-mail: mazor@math.uu.se 